ARTICLE

DOI: $10.1038 / s 41467-018-06908-5$

\title{
A distinct isoform of ZNF207 controls self-renewal and pluripotency of human embryonic stem cells
}

\author{
Fang Fang (1) 1,2, Ninuo Xia ${ }^{1,2}$, Benjamin Angulo ${ }^{1,2}$, Joseph Carey ${ }^{1,2}$, Zackery Cady ${ }^{1,2}$, Jens Durruthy-Durruthy ${ }^{3}$,
} Theo Bennett ${ }^{1,2}$, Vittorio Sebastiano ${ }^{3} \&$ Renee A. Reijo Pera (i) ${ }^{1,2}$

Self-renewal and pluripotency in human embryonic stem cells (hESCs) depends upon the function of a remarkably small number of master transcription factors (TFs) that include OCT4, SOX2, and NANOG. Endogenous factors that regulate and maintain the expression of master TFs in hESCs remain largely unknown and/or uncharacterized. Here, we use a genome-wide, proteomics approach to identify proteins associated with the OCT4 enhancer. We identify known OCT4 regulators, plus a subset of potential regulators including a zinc finger protein, ZNF207, that plays diverse roles during development. In hESCs, ZNF207 partners with master pluripotency TFs to govern self-renewal and pluripotency while simultaneously controlling commitment of cells towards ectoderm through direct regulation of neuronal TFs, including OTX2. The distinct roles of ZNF207 during differentiation occur via isoform switching. Thus, a distinct isoform of ZNF207 functions in hESCs at the nexus that balances pluripotency and differentiation to ectoderm.

\footnotetext{
${ }^{1}$ Department of Cell Biology and Neurosciences, Montana State University, Bozeman, MT 59717, USA. ${ }^{2}$ Department of Chemistry and Biochemistry, Montana State University, Bozeman, MT 59717, USA. ${ }^{3}$ Department of Genetics, Institute for Stem Cell Biology and Regenerative Medicine, Stanford University School of Medicine, Stanford, CA 94305, USA. These authors contributed equally: Fang Fang, Ninuo Xia. Correspondence and requests for materials should be addressed to F.F. (email: fangfang0724@gmail.com)
} 
$\mathrm{H}$ uman embryonic stem cells (hESCs) possess the capacity to renew indefinitely (self-renewal) while maintaining the potential to differentiate into any somatic cell types (pluripotency). Self-renewal and pluripotency are regulated by a unique transcriptional network controlled by a small number of endogenous master transcription factors (TFs) that include OCT4, SOX2, and NANOG ${ }^{1-5}$. Disruption in the expression of critical TFs leads to loss of pluripotency and commitment of cells to differentiate into diverse cell lineages. For example, in mouse embryonic stem cells (mESCs), a 50\% reduction in expression of OCT4 (also known as POU5F1) triggers mESC differentiation to trophoblasts; conversely, a twofold increase in expression promotes differentiation to primitive endoderm and mesoderm ${ }^{6}$. While multiple reports document the function of TFs via studies that modulate exogenous expression in pluripotent stem cells, much less is known about how TFs are endogenously regulated.

Recently, locus-specific proteomics makes use of the genomic targeting ability of the TALEN (Transcription Activator-Like Effector Nuclease) or CRISPR (Clustered Regularly Interspaced Palindromic Repeats) systems to achieve high resolution and specific isolation of factors that bind native genomic loci. It has the potential to provide insight into endogenous protein and epigenetic mechanisms that regulate gene expression ${ }^{7-14}$. However, these studies have never been done in hESCs.

Our study reports the adaptation and optimization of locusspecific proteomics to target the regulatory element of the OCT4 gene in hESCs. It is important to note that OCT4 gene expression is regulated by three regulatory elements: a distal enhancer (DE), a proximal enhancer (PE), and a proximal promoter (PP) ${ }^{15-17}$. The PE element is used in hESCs to maintain OCT4 expression ${ }^{18}$. The goal of this study was to identify nuclear proteins bound at the PE of OCT4, including most known OCT4 regulators as well as TFs that have not previously been shown to bind at this locus. Among the list of TFs, we identified a zinc finger protein, ZNF207. Zinc finger-containing proteins (ZNFs) are the largest transcription factor family in the human genome, where, in combination with other TFs or co-factors, ZNFs play diverse roles in development, differentiation, and metabolism. This study reports identification and characterization of the essential roles of ZNF207 in self-renewal, pluripotency, and differentiation.

\section{Results}

Locus-specific proteomics identified OCT4 regulators. We set out to develop an unbiased, genome-wide method to screen for proteins that locate to the OCT4 PE in hESCs; for this purpose, we developed an optimized locus-specific proteomics approach in hESCs (Fig. 1a). First, we designed TALEN constructs to target the sequences that are near the OCT4 PE, located in a region of DNaseI hypersensitivity (Supplementary Fig. 1a). TALEN constructs with the highest cutting efficiency were chosen for locusspecific proteomics (Supplementary Table 1). We then made modifications to the original TALEN protein to transform it into a catalytically-dead TALE (dTALE) protein that is optimized for locus-specific proteomics in hESCs (Supplementary Fig. 1b) via three steps: (1) The nuclease-domain FokI at the C-terminus was replaced by a GFP (green fluorescence protein); (2) a 3X FLAG tag at the $\mathrm{N}$-terminus was included for following pull-down analysis; (3) the existing CMV promoter was replaced with an EF1a promoter that has robust expression in hESCs (Supplementary Fig. 1c). This dTALE protein could then be chemically crosslinked to the OCT4 locus together with all the other proteins that bind to the locus. We verified that dTALE protein binds to the targeted locus by chromatin immunoprecipitation (ChIP)qPCR (Fig. 1b). Following crosslinking, chromatin was sheared, and all the associated proteins were immunoprecipitated using an
anti-FLAG antibody. Immunoprecipitation pulled down the dTALE protein (Supplementary Fig. 1d) as well as other proteins and complexes that are also attached to that region (Supplementary Fig.1e). Crosslinking was then reversed, and the samples were subjected to mass spectrometry to enable generation of a list of proteins that potentially bind to OCT4 PE locus.

Mass spectrometry resulted in the identification of 150 nucleus proteins co-immunoprecipitated with dTALE protein (Supplementary Data file). The list of proteins includes proteins that are known to bind to and regulate OCT4, including Sex Determining Region Y-Box 2 (SOX2), Spalt-Like Transcriptional Factor 4 (SALL4), Histone Deacetylase 2 (HDAC2), and OCT4 itself

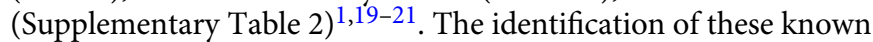
regulators of OCT4 provides support for the reliability of our approach. In addition to these known OCT4 locus-bound proteins, mass spectrometry also generated a list of nuclear proteins, including a few TFs (Supplementary Table 3). Enriched Gene Ontology (GO) categories for these proteins were identified using DAVID (the Database for Annotation, Visualization and Integrated Discovery) (Fig. 1c). These proteins include those that are involved in transcriptional regulation, translational regulation, nuclear structure, as well as, nuclear transport machinery. This is consistent with the previous findings that the transcriptional machinery is coupled with the translational machinery and nuclear transport machinery at transcriptionally active sequences $^{22}$.

ZNF207 is required for self-renewal and pluripotency. Among the 150 nuclear proteins identified, we focused on TFs and identified a subset for further verification (Supplementary Table 3); all of these demonstrated binding at the PE of OCT4 by ChIP-qPCR (Supplementary Fig. 2a). OCT4 protein was used as the positive control. To test whether these TFs play regulatory roles at the OCT4 genetic locus, we performed siRNA-mediated knockdown (KD) analysis. Q-PCR results indicated that KD of several of these TFs led to a significant change in OCT4 mRNA expression. KD of $M B D 3, C B X 3, I G F 2 B P 1$, and OCT4 caused reduction of OCT4 expression, while KD of HDAC2 and SALL2 resulted in upregulation of OCT4 expression (Supplementary Fig. 2b). Both downregulation and upregulation of OCT4 in hESCs results in differentiation. These results, consistent with the published data, suggest that OCT4 level must be tightly controlled in order to maintain self-renewal and pluripotency ${ }^{6,23}$. These results also demonstrated the ability of the optimized locusspecific proteomics method to identify putative regulators of OCT4 that may function in maintaining self-renewal and pluripotency of hESCs.

The KD of ZNF207 was notable as it triggered a dramatic morphological change in hESC colonies, even relative to the $\mathrm{KD}$ phenotype of OCT4 and SOX2 (Fig. 2a). The mRNA and protein levels of ZNF207 were reduced by siRNAs to about $5-10 \%$ (Supplementary Fig. 2c). The morphological changes were correlated with loss of alkaline phosphatase (AP) staining (Fig. 2b). Utilizing ChIP, we also verified that ZNF207 binds to the PE of OCT4 (Fig. 2c). As ZNF207 expression decreased, protein expression of OCT4 and NANOG was drastically reduced; moreover, the hESC surface marker TRA-1-60, changed its localization from even distribution on the cell membrane to a focal point inside the cell (Fig. 2d). These results indicated that hESCs have undergone dramatic changes, commonly associated with loss of pluripotency and self-renewal, in response to reduced expression of ZNF207. To further examine the role of ZNF207 in induction of the endogenous OCT4 gene, we compared the colony formation efficiency during the reprogramming process from human neonatal fibroblast to induced pluripotent stem cells 
a

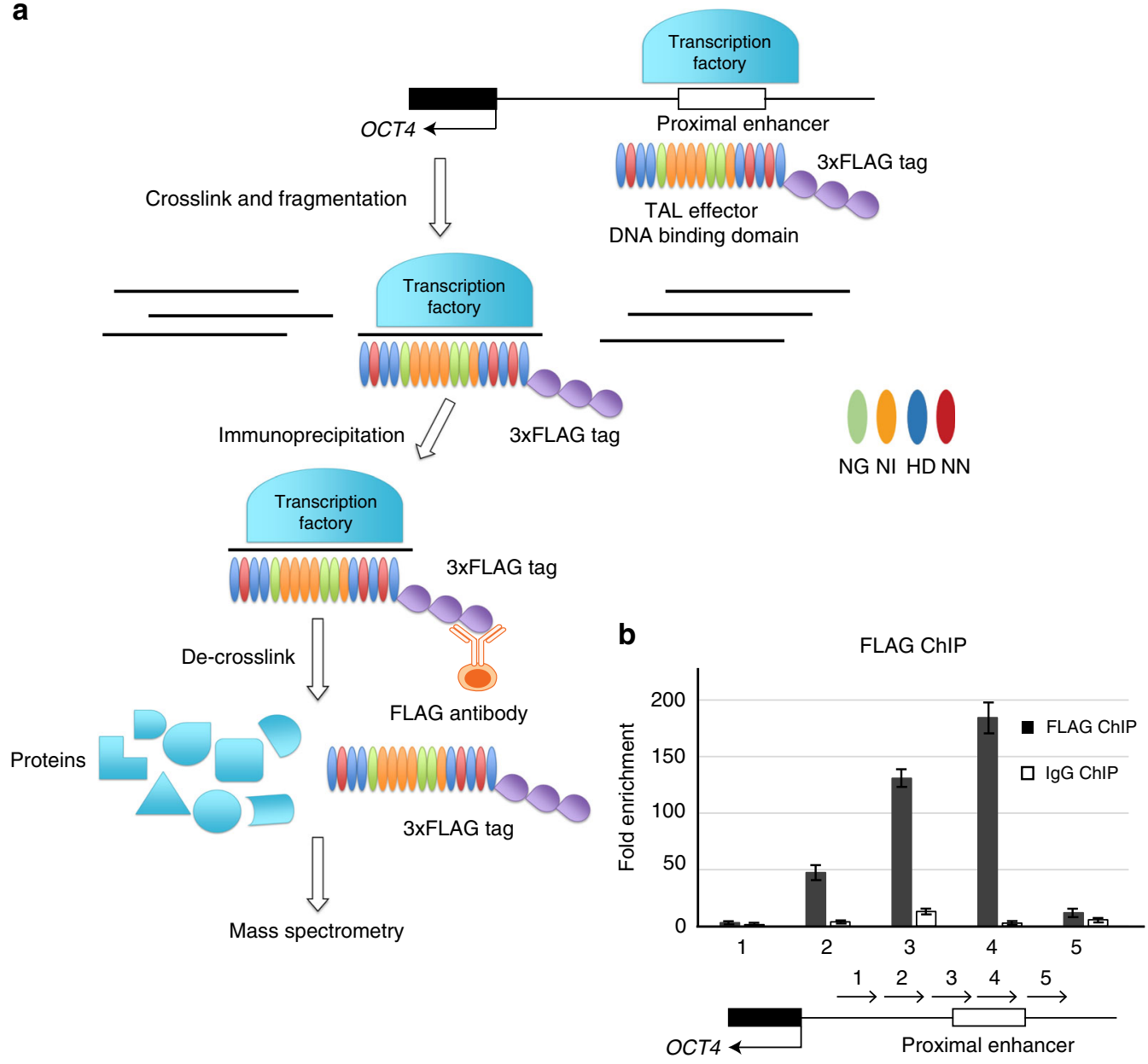

C 0.00E+00 2.00E-05 4.00E-05 6.00E-05 8.00E-05 1.00E-04 P-value

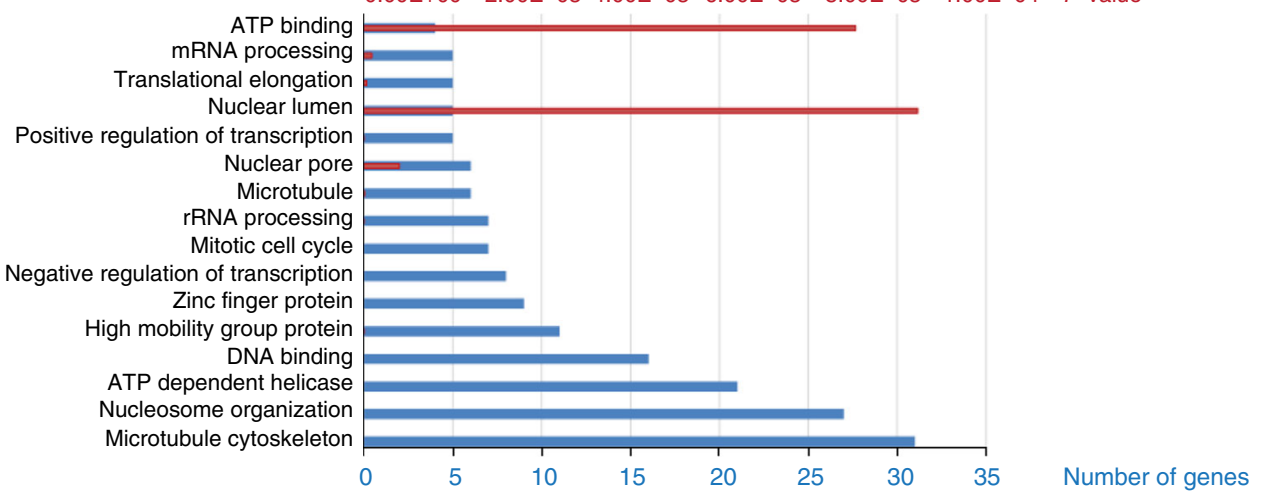

Fig. 1 Locus-specific proteomics identified proteins located at the proximal enhancer of OCT4 gene in hESCs. a Schematic overview of locus-specific proteomics in hESCs. A representation of OCT4 locus is shown on the top. Dark boxes represent exons, and the white box represents the proximal enhancer that is bound by the transcription factory. TALEN protein with a 3xFLAG tag was designed to bind to the proximal enhancer. The colored ovals represent the "repeat-variable di-residues" (RVD) of TALEN protein that determines binding specificity to DNA bases. They follow the code that NG, NI, $H D$, and NN respectively recognizes thymine, adenine, cytosine, and guanine. Chromatin is crosslinked by formaldehyde and fragmented by sonication. Then, FLAG antibody was used for immunoprecipitation of proteins bound to the proximal enhancer. The pull-down complex was de-crosslinked of the identity of isolated proteins was discovered by mass spectrometry. $\mathbf{b}$ Validation of the binding of dTALE protein by ChIP. A ChIP assay was performed using anti-flag antibody to detect enriched fragments in hESCs. Fold enrichment is the relative abundance of DNA fragments at the amplified region over a control amplified region. IgG ChIP is served as control. The locations of the amplified products are indicated by arrows along the proximal promoter of OCT4. Data are presented as the mean \pm SEM and are derived from three independent experiments. c Gene ontology analysis of the proteins identified by locus-specific proteomics. Blue bars represent the number of genes and red bars represent $p$-value. $p$-value is calculated using hypergeometric distribution 
a
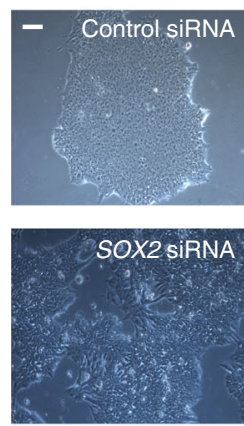
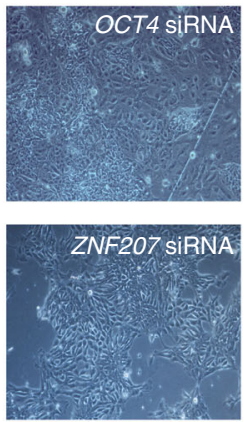

b
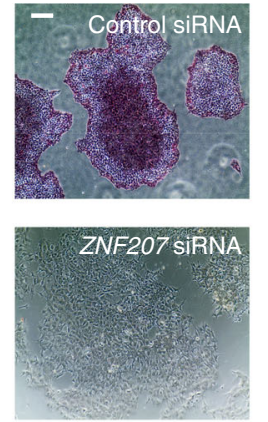

C

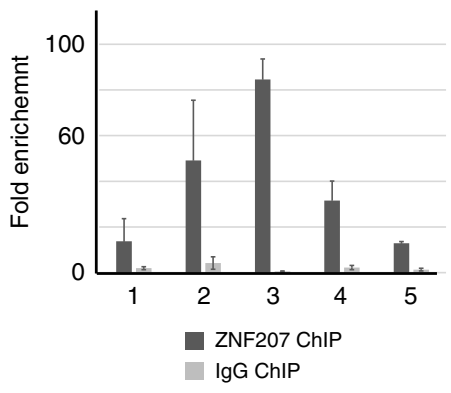

d

ZNF207/DAPI

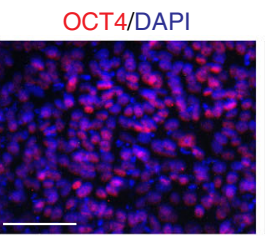

NANOG/DAPI

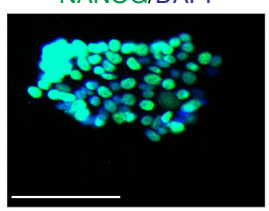

TRA1-60/DAPI
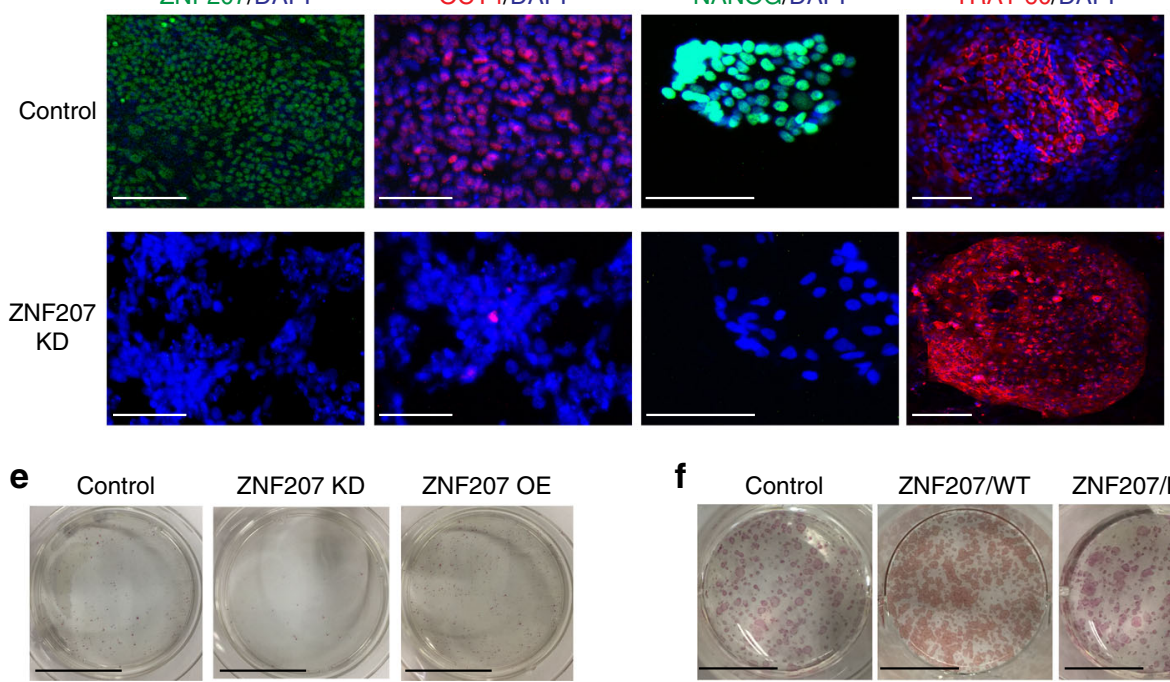

f
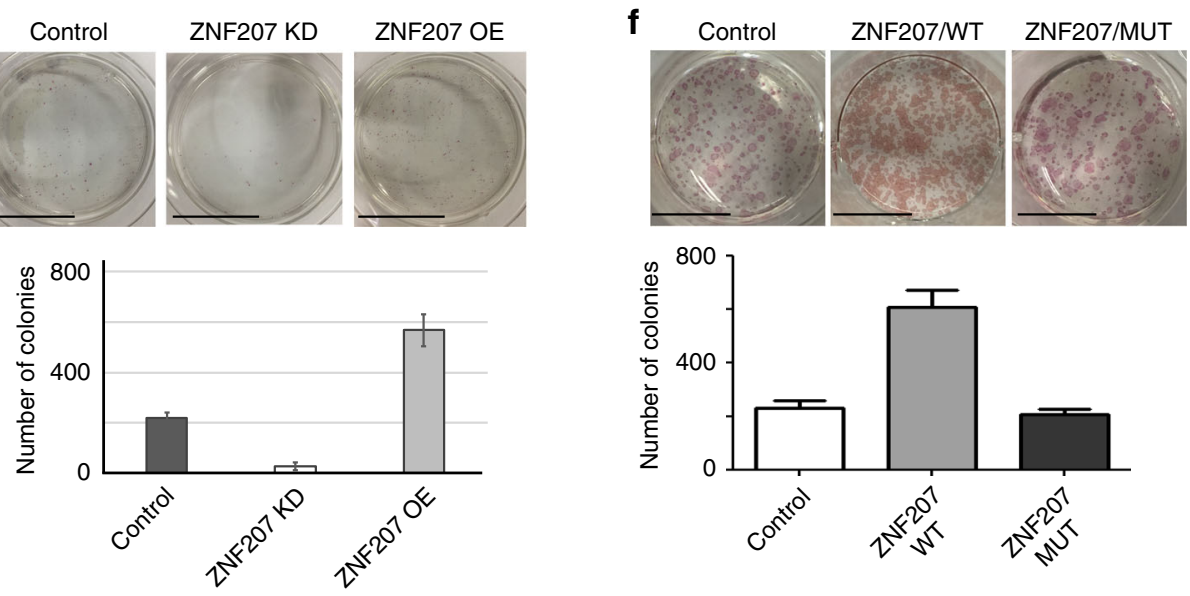

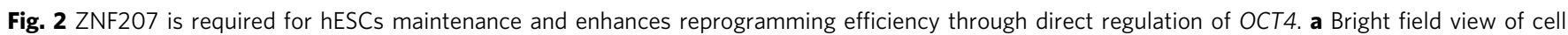
morphology after knockdown. Scale bars represent $50 \mu \mathrm{m}$. b Alkaline phosphatase staining of cells after knockdown. Scale bars represent $50 \mu \mathrm{m}$. c Validation of the binding of ZNF207 by ChIP. A ChIP assay was performed using anti-ZNF207 antibody to detect enriched fragments in hESCs. IgG ChIP is served as control. The locations of the amplified products are labeled as Fig. 1b. d Immunofluorescence staining of control and ZNF207 KD hESCs. Scale bars represent $50 \mu \mathrm{m}$. e Overexpression of ZNF207 (ZNF207 OE) enhanced reprogramming efficiency, while knockdown of ZNF207 (ZNF207 KD) reduced the efficiency. Top: Colony formation shown by AP staining. Bottom: Counts of colonies. Scale bars represent $5 \mathrm{~cm}$. $\mathbf{f}$ Deletion of ZNF207 DNAbinding domain abolished its effect on reprogramming. Top: Colony formation shown by AP staining. Bottom: Counts of colonies. Scale bars represent

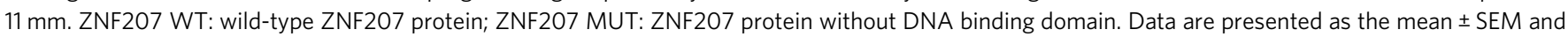
are derived from three independent experiments

(iPSCs). Strikingly, we found that KD of ZNF207 during the process of reprogramming greatly reduced the efficiency of reprogramming by about tenfold, while overexpression of ZNF207 enhanced the efficiency by about threefold (Fig. 2e). Note that the change in reprogramming efficiency does not appear to be directly linked to alteration of cell proliferation; the KD of ZNF207 resulted in a decrease in the cell proliferation rate to approximately $75 \%$ of that of the control, whereas, OE of ZNF207 had no obvious effects on cell proliferation (Supplementary Fig. 2d). We observed higher expression of endogenous OCT4 in ZNF207 OE cells and lower expression of endogenous
OCT4 in ZNF207 KD cells, which correlates with the reprogramming efficiency in each condition (Supplementary Fig. 2e). To better understand the molecular mechanism of ZNF207 in promoting the reprogramming efficiency, we mutated ZNF207 protein by deleting its DNA binding domain (ZNF207/MUT) and introduced it to the reprogramming process, in comparison to the wild-type ZNF207 protein OE (ZNF207/WT) and no ZNF207 OE (Control) conditions. We found that mutated ZNF207 is not able to promote increased reprogramming efficiency (Fig. 2f). This result suggests that ZNF207 promotes reprogramming by regulation of gene transcription through its DNA binding 
domain. Taken together, these results indicate that ZNF207 is essential for the maintenance of hESCs and reprogramming of human pluripotent stem cells from somatic cells.

Recently, it has been demonstrated that there are at least two distinct pluripotent states, termed the primed and naive states. hESCs and human-induced pluripotent stem cells (hiPSCs) are most similar to the post-implantation epiblast, whereas, naivestate human pluripotent stem cells (hPSCs) are more comparable to the preimplantation epiblast ${ }^{24}$. To determine whether ZNF207 functions in naive-state hPSCs as well as primed, we performed knockdown analysis in naive hPSCs. The naive human pluripotent stem cells were derived and maintained based on the protocol of Takashima et al. ${ }^{25}$ We observed that depletion of ZNF207 in naive-state hPSCs resulted in disruption of colony forming morphology (Supplementary Fig. 3a), loss of alkaline phosphatase staining (Supplementary Fig. 3b), reduced expression of pluripotency genes, and upregulation of lineage marker genes (Supplementary Fig. 3c). These results suggested that ZNF207 may be a critical regulator in human embryo development; thus, we examined whether ZNF207 is expressed in preimplantation human embryos. We observed that ZNF207 is activated at the 8-cell stage, with timing similar to many other factors including NANOG (Supplementary Fig. 3d). The expression of ZNF207, however, was significantly higher than NANOG across all the stages of preimplantation development (Supplementary Fig. $3 e^{26,27}$.

Identification of direct ZNF207 target genes in hESCs. To investigate the global regulatory roles of ZNF207 in hESCs, RNASeq was used to compare the transcriptomes in ZNF207 KD cells and control hESCs. A total of 1287 genes were found to be downregulated and 785 genes were upregulated by at least twofold in ZNF207 KD cells compared to control (Fig. 3a). To establish which of the genes, that were differentially expressed between KD and control cells, are directly regulated by ZNF207, ChIP-Seq was used to profile the genome-wide binding sites of ZNF207 in hESCs. Approximately 8000 ZNF207 binding sites were identified across the genome of hESCs, with the strongest binding clustered around the transcription start site (TSS) (Fig. 3b). Note that a previously unknown motif of statistical significance for ZNF207 was identified and direct binding of ZNF207 protein to this DNA sequence was confirmed by electrophoretic mobility shift assays (EMSA). Mutation of specific conserved nucleic acids in the motif abolished the binding of the ZNF207 protein to the DNA sequences (Fig. 3c, d).

ChIP-Seq confirmed that ZNF207 binds to OCT4 PE, as well as $\mathrm{DE}$, and it co-localizes with master TFs, OCT4, SOX2, and NANOG, at this locus (Fig. 3e). Its direct regulation of transcription through the OCT4 PE was confirmed by luciferase reporter assays (Supplementary Fig. 4a). Furthermore, the effects of ZNF207 KD were rescued by overexpression of OCT4 (Supplementary Fig. 4b, c), suggesting that OCT4 is a major downstream target of ZNF207 in hESCs. Taken together, these data indicate that OCT4 is one of the direct and major functional targets of ZNF207 in hESCs and in the process of reprogramming from somatic cells to pluripotent cells.

In addition to OCT4, we were interested in identification of downstream targets of ZNF207 in the whole genome of hESCs. We defined that genes that are bound by ZNF207 and differentially expressed in ZNF207 KD cells as direct ZNF207 targets in hESCs. Thus, 177 direct target genes that were upregulated and 357 genes that were downregulated in response to ZNF207 KD were identified (Fig. 3f). The GO (Gene Ontology) terms of ZNF207 negatively correlated genes (upregulated in ZNF207 KD cells) were related to cellular processes such as programmed cell death, cardiovascular development, angiogenesis, and cholesterol biosynthesis (Fig. 3g, top panel), suggesting the possibility that ZNF207 may play a role in repression of apoptosis and mesoderm development. GO terms of ZNF207 positively correlated genes (downregulated in ZNF207 KD cells) were related to cellular process such as cell proliferation, neurogenesis, and beta-catenin-TCF complex assembly (Fig. 3g, bottom panel), suggesting that ZNF207 may function in cell cycle regulation, ectoderm development, and stem cell signaling pathways.

ZNF207 is a part of the OCT4/SOX2 core pluripotency network. To probe its role in pluripotency, we analyzed the in vivo sequence specificity of ZNF207. Interestingly, the OCT4/SOX2 consensus motif was enriched as the most significant motif with an E-value of 5.3E - 059 (Fig. 4a) and we observed overlap of OCT4, SOX2, and ZNF207 binding events (Fig. 4b). This reflects the frequent co-localization of ZNF207 with OCT4/SOX2 across the genome of hESCs and suggests that ZNF207 could be one of the interaction partners of the OCT4/SOX2 core pluripotency network. To test potential protein-protein interactions, we performed co-immunoprecipitation (Co-IP) experiment using hESC cell extracts. We detected ZNF207 protein in OCT4 and SOX2 pull-down and vice versa (Fig. 4c), indicating that ZNF207 forms a complex with OCT4/SOX2 in hESCs through protein-protein interactions. In addition to the OCT4/SOX2 motif, we identified known motifs for neuronal TFs, such as ZIC1/ZIC2, SOX10/ SOX3/SOX11, TCF3, and ASCL1 (Supplementary Fig. 4d), suggesting that ZNF207 may play a role in neuronal development upon differentiation.

We then analyzed genes that were co-bound by ZNF207, OCT4, and SOX2 (Fig. 4d) and observed that these genes are enriched in the GO terms of embryo development, ectoderm development, and nerve system development (Fig. 4e). For example, these genes include critical pluripotency marker genes, such as OCT4, SOX2, SALL4, NANOG, and ZNF207 itself (Fig. 4f), and important neuroectoderm developmental genes such as NES, NEUROG3, NCAM1, and GLI2 (Fig. 4g). Taken together, our data strongly suggested that ZNF207 is part of the OCT4/SOX2 core pluripotency complex and they collaboratively target the pluripotency and ectoderm developmental genes in hESCs.

ZNF207 promotes self-renewal of hESCs. We observed cooccurrence of ZNF207 with enhancer markers, P300 and H3K27ac in hESCs (Fig. 5a). Apart from "stem cell maintenance genes" that are also co-bound by OCT4/SOX2 (Supplementary Fig. 5a), genes co-marked by ZNF207/P300/H3K27ac largely belong to categories related to cell cycle processes (Fig. 5b). Individual genes, CDK1, BUB1, TERF1, and TRIAP1, for examples, are heavily bound by ZNF207 and enhancer markers, P300 and H3K27ac (Fig. 5c). KD of ZNF207 led to differential expression of a few genes involved in hESCs proliferation (Supplementary Fig. 5b). We found that genes involved in senescence, cell cycle checkpoint and cell cycle phase transition were upregulated, and genes involved in regulation of cyclin-dependent protein kinase $(\mathrm{CDK})$ activity, telomerase activity and DNA damage response were downregulated in ZNF207 KD cells (Fig. 5d). This is consistent with the findings that pluripotent cells exhibit high CDK and telomerase activity, lack checkpoint regulation and do not appear to undergo senescence. When differentiation occurs or pluripotency is lost, cells become more cell cycle regulated and their DNA repair ability is decreased ${ }^{28-30}$. We also confirmed that the change in expression of RNAs of cell cycle genes were reflected in protein levels (Supplementary Fig. 5c). Further cell cycle analysis demonstrated that reduced levels of 
a

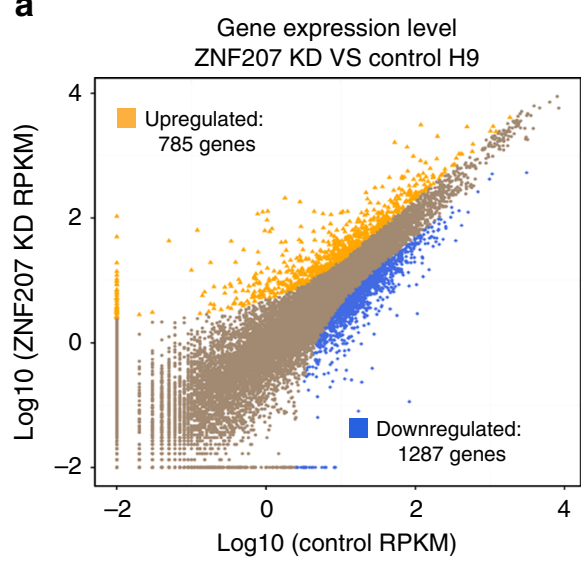

C

E-value: $3.6 \mathrm{E}-030$

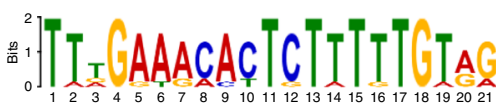

TTTGAAACACTCTTTTTGTAG WT

TTTTAAACACGCGTGTGTTAG MUT

$\mathbf{f}$
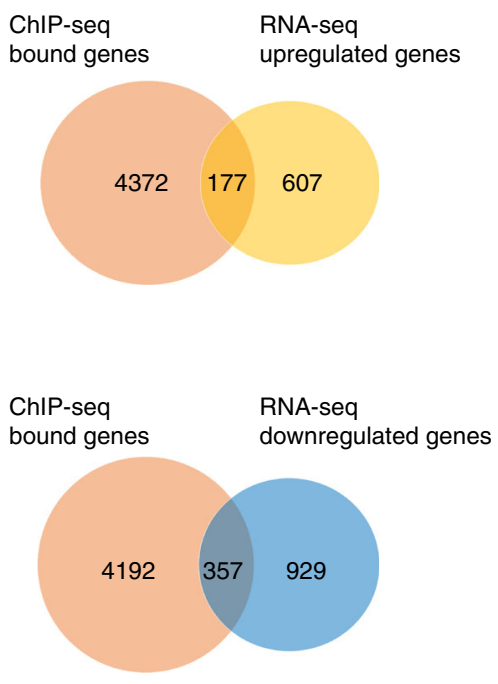

b

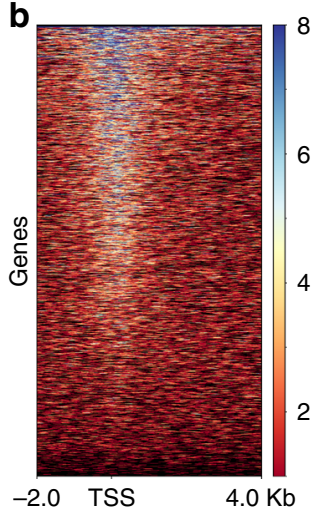

6

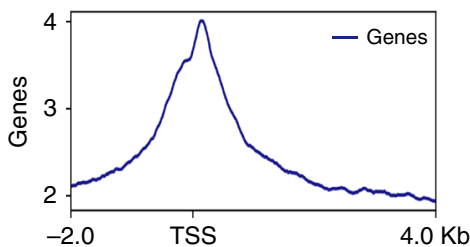

e

ZNF207

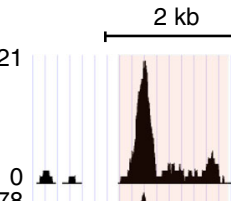

d

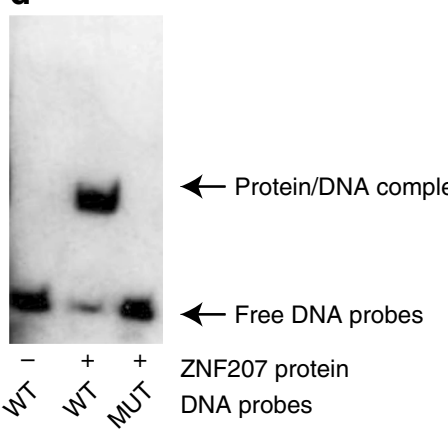

0
78

sox2

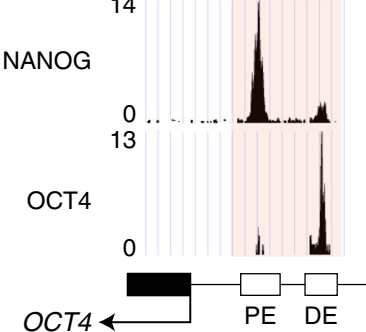

g
Upregulated genes

0

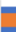

20
40

60

Positive regulation of programmed cell death

Cardiovascular system development

Intrinsic apoptotic signaling pathway

Sprouting angiogenesis

Alcohol biosynthetic process

Cholesterol biosynthetic process

Endopeptidase activity involved in apoptosis

$$
p \text {-value }
$$

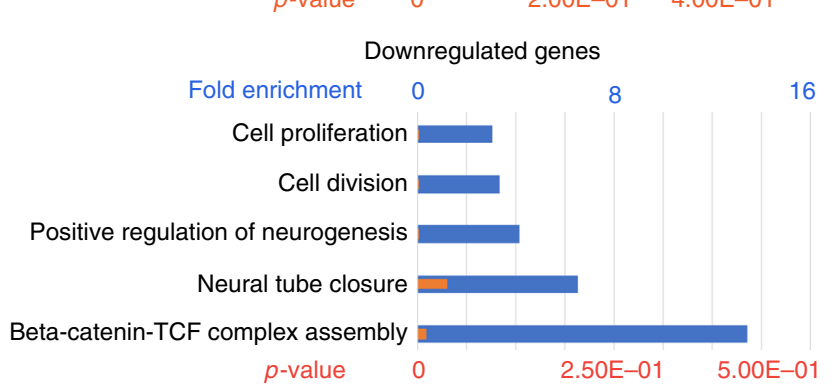

Fig. 3 High-throughput analysis in hESCs to identify direct targets of ZNF207. a Analysis of differential expression on the RNA-Seq data of ZNF207 KD and control hESCs. Genes significantly changed (>twofold change, FDR $=1 \%$ ) are colored in yellow and blue for upregulated and downregulated, respectively. b ZNF207 ChIP-Seq analysis in hESCs. Left: Heatmap depicts ZNF207 ChIP-Seq signals at TSSs. Right: Composite plot shows ZNF207 ChIP-Seq signal is enriched at transcription start sites (TSSs). The gradient blue-to-red color indicates high-to-low counts in the corresponding region. $\mathbf{c}$ Enriched motif from de novo motif search of sequences under ZNF207 peaks. Wild-type (WT) sequence and mutant (MUT) sequence are shown in the bottom. Mutated nucleic acids are labeled by red fond color. d EMSA were performed to detect interaction of ZNF207 protein with DNA sequences. e ChIP-Seq tracks show co-localization of ZNF207, SOX2, NANOG, and OCT4 at the proximal enhancer of OCT4 gene. OCT4 gene, Proximal enhancer (PE) and distal enhancer $(\mathrm{DE})$ are indicated by the boxes in the bottom. The scale bar indicates the size of the chromosome. The light pink boxes highlight the co-bound regions. f Venn diagrams show overlaps of ChIP-Seq bound genes with differentially expressed genes identified from RNA-Seq. $\mathbf{g}$ Gene ontology analysis of genes that are directed regulated by ZNF207. Blue bars represent fold enrichment and orange bars represent $p$-value. $p$-value is calculated using hypergeometric distribution 
a

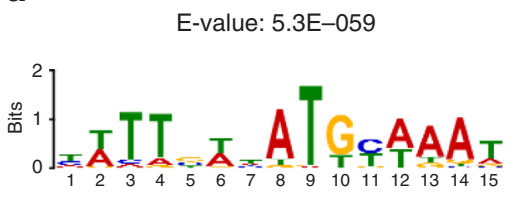

b z

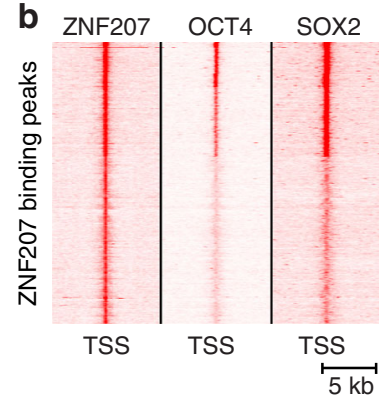

d

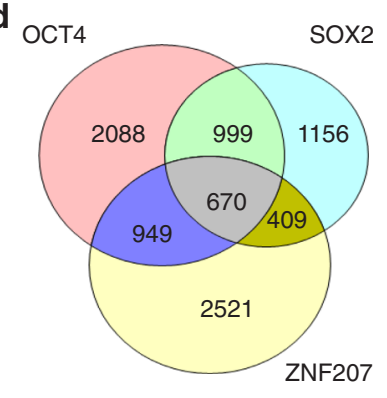

C

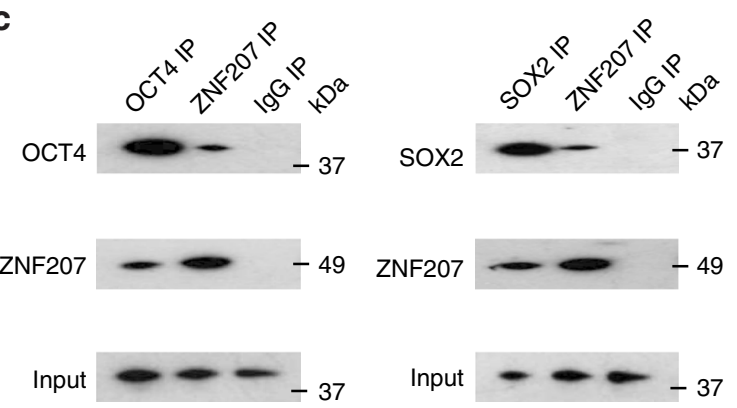

f

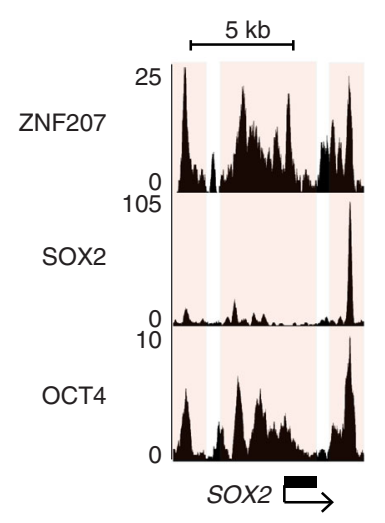

g

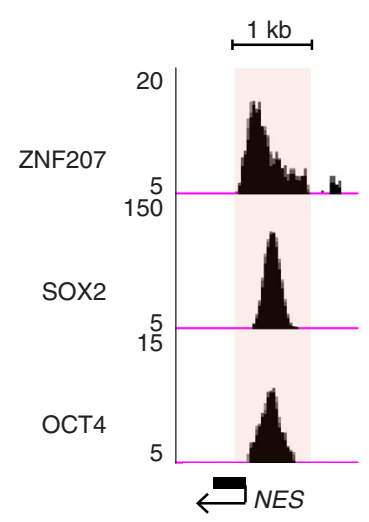

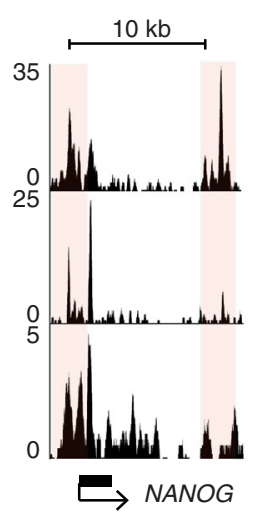

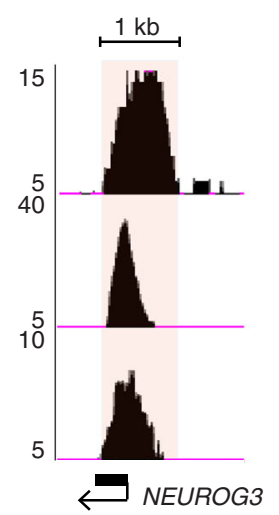

e

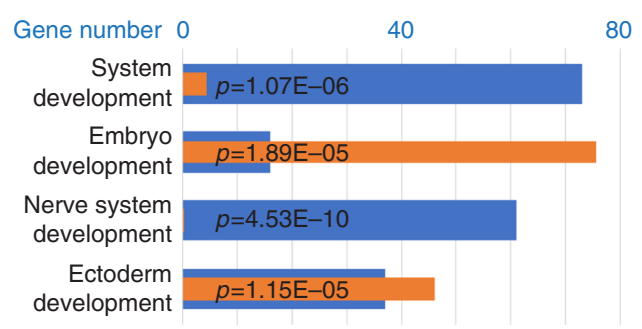

$p$-value $0.00 \mathrm{E}+00 \quad 1.00 \mathrm{E}-05 \quad 2.00 \mathrm{E}-05$
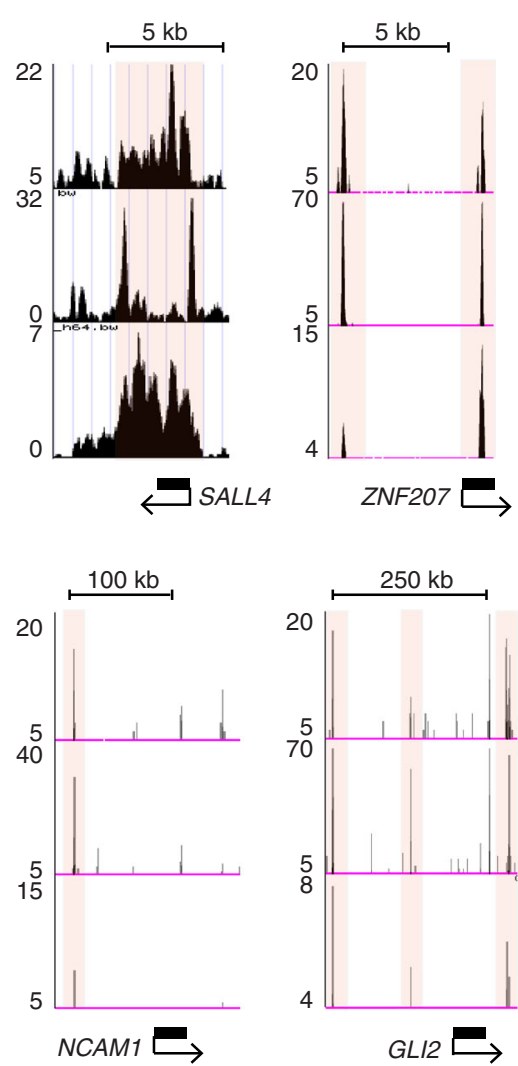

ZNF207 inhibits proliferation of hESCs (Supplementary Fig. 5d) by decreasing the percentage of cells in $\mathrm{S}$ and $\mathrm{G} 2 / \mathrm{M}$ phase and increasing the percentage of cells in G0/G1 phase (Fig. 5e). These findings suggest that ZNF207 is required for cell cycle progression of hESCs. Reduced cell cycle progression and the lengthening of G0/G1 phase may provide a window for differentiation signals.

More interestingly, we found that FGF2 (bFGF) could be one of the direct targets of ZNF207: ZNF207 binds to the promoter and enhancer regions of FGF2 gene (Fig. 5f). The mRNA and protein expression levels of bFGF were correlated with the levels of ZNF207 (Fig. 5g, h). It has been shown that bFGF signaling is of central importance to hESCs self-renewal ${ }^{31-34}$. Thus, we used clonogenicity assays to test whether ZNF207 OE could prolong self-renewal of hESCs in the absence or low concentrations of bFGF. The data indicated that control hESCs failed to survive in $20 \mathrm{ng} / \mathrm{ml}$ bFGF culture media, whereas, ZNF207 OE hESCs could proliferate as single cells and form AP-staining positive colonies (Fig. 5i). These results suggest that ZNF207 OE promotes the 
Fig. 4 ZNF207 co-localizes with master transcription factors in hESCs to regulate pluripotency and neuronal gene expression. a Enriched motifs from de novo motif search of sequences under ZNF207 peaks. Note the identification of consensus OCT4/SOX2 binding but also other known transcription factor binding motifs. Statistical significance (E-values) is indicated below the motif logo. b Heatmap depicts ZNF207, OCT4 (GSM1124067), and SOX2 (GSM1701825) ChIP-Seq signals at TSSs. c Protein interactions between ZNF207 with OCT4 and SOX2. Co-IP using cell extracts from hESCs was performed using anti-OCT4 and anti-SOX2 antibody. Western blotting was carried out with anti-ZNF207 antibody. Control IP was performed using antiIgG antibody. Reverse co-IP was performed using anti-ZNF207 antibody, and western blotting was then performed with anti-OCT4 or anti-SOX2 antibody. Input was shown as loading control. d Venn diagrams show overlaps of genes bound by OCT4, SOX2, and ZNF207 in hESCs. e Gene ontology analysis of genes that are directed regulated by ZNF207. Blue bars represent the number of genes and orange bars represent $p$-value. $p$-value is calculated using hypergeometric distribution. $\mathbf{f}$ ChIP-Seq tracks show co-localization of ZNF207, SOX2, and OCT4 at the regulatory sequences of pluripotency genes. The scale bars indicate the size of the chromosome. The light pink boxes highlight the co-bound regions. $\mathbf{g}$ ChIP-Seq tracks show co-localization of ZNF207, SOX2, and OCT4 at the regulatory sequences of neuronal genes. The scale bars indicate the size of the chromosome. The light pink boxes highlight the cobound regions

production of bFGF from the cells, prolonging self-renewal of hESCs in low concentrations of bFGF.

ZNF207 directs specification to ectoderm. Previous studies suggested that the core pluripotent TFs, OCT4, SOX2, and NANOG may not only play important roles in the induction and maintenance of pluripotency, but may also function as lineage specifiers, regulating the differentiation of ESCs to specific lineages ${ }^{35-37}$. Although high-throughput and loss-of-function studies shed light on ZNF207 as a part of the core pluripotency network that maintains the identity of hESCs, its role in regulating differentiation was not clear. To investigate the differentiation potential of ZNF207 KD hESCs, cells were differentiated in vitro into the three germ layers: ectoderm, mesoderm, and endoderm. ZNF207 siRNAs were transfected into the hESCs every 2 days to ensure that the levels of ZNF207 were constantly reduced during differentiation (Supplementary Fig. 6a). Results of immunostaining revealed higher expression levels of endodermal (SOX17/FOXA2) and mesodermal (NCAM) marker proteins in ZNF207 KD cells and reduced expression of ectodermal marker (NES) proteins compared to control H9 cells (Fig. 6a). To quantify, we counted the total number and the percentage of cells that stained positive for each lineage and found that ZNF207 KD cells were able to differentiate more efficiently to endodermal and mesodermal cells, and less so to ectodermal cells (Fig. 6b). These results indicated that $\mathrm{KD}$ of ZNF207 in hESCs disrupts the balance of pluripotency and differentiation (Fig. 6c).

Notably, we observed that OTX2, an early ectodermal lineage marker gene, is bound by ZNF207 (Supplementary Fig. 6b). In the process of ectoderm differentiation from hESCs, OTX2 expression is induced to levels that facilitate further differentiation. In ZNF207 KD cells, OTX2 expression cannot be induced efficiently and is at much lower level compared to the control. However, its expression is restored by a rescue experiment with ZNF207 OE (Supplementary Fig. 6c). These data indicate that OTX2 is a direct target of ZNF207. To address the possibility that reduced expression of OTX2 might be linked to the deficiency in ectoderm differentiation, we overexpressed OTX2 in ZNF207 KD hESCs to the comparable level of control cells (Supplementary Fig. 6d) to test whether this could rescue differentiation to ectoderm. Indeed, overexpression of OTX2 restored the ability of ZNF207 KD cells to differentiate to ectoderm as detected via reexpression of early ectoderm markers, NES and SOX2 (Fig. 6d). We also observed upregulation of FOXG1 and NGN2, which are early neuroectoderm markers that work downstream of OTX2 (Supplementary Fig. 6d). Quantification analysis showed increased number of ectoderm cells under rescue conditions (ZNF207 KD, OTX2 OE) compared to ZNF207 KD (Fig. 6e). Thus, ZNF207 is part of the core pluripotency network and forms complexes with OCT4/SOX2/P300. It safeguards pluripotency by directly controlling key pluripotency genes and maintaining cell proliferation and self-renewal by activating cell cycle genes. In addition, ZNF207 ensures ectoderm differentiation potential by maintaining expression of early ectoderm genes, such as OTX2. KD of ZNF207 causes reduced expression of pluripotency genes, compromises stem cell self-renewal, and blocks ectoderm differentiation (Fig. 6f).

ZNF207 switches isoforms during cell differentiation. ZNF207 was originally discovered as a kinetochore-binding protein that promotes spindle assembly during mitosis in HeLa cells and Glioblastoma multiforme stem cells (GSCs) ${ }^{38-40}$ Whether ZNF207 also controls spindle assembly in hESCs is still unknown. To investigate, we knocked down ZNF207 and assessed whether it causes defects in spindle formation as reported in cancer cells (Supplementary Fig. 7a, b). Kinetochores were stained with CREST (centromere) antibodies, microtubules were stained with an anti-a-tubulin antibody, and chromosomes were stained with DAPI. To our surprise, we did not observe much misalignment in mitotic cells in both control and ZNF207 KD hESCs (Fig. 7a). We also quantified the percentage of misaligned chromosomes and found only $0-1 \%$ of the cells were misaligned and there was no significant difference between control and KD cells (Fig. 7b).

The data above provided substantial evidence that ZNF207 functions as a critical TF in hESCs to regulate downstream pluripotency and developmental gene expression; a role in regulating mitotic chromosome alignment was not observed. Previous studies indicated that ZNF207 has three isoforms (isoform $\mathrm{A}, \mathrm{B}$, and $\mathrm{C}$ ). Isoform $\mathrm{C}$ is the longest isoform; isoform A lacks exon 6, and isoform B lacks exon 9 (Fig. 7c). ZNF207 mRNA is the target of a splicing factor SFRS11 and during somatic reprogramming, ZNF207 changes from isoform B to A and $\mathrm{C}^{41}$. Thus, we investigated whether different functions of ZNF207 are linked to the presence of various isoforms in different cell types. We note that we used isoform C for all the ZNF207 protein experiments, including EMSA (Fig. 3e) and overexpression (Figs. 2e, f and 5h, i) analysis. We initiated neuronal differentiation of hESCs and found that ZNF207 gradually switches its isoform from $\mathrm{A}$ and $\mathrm{C}$ to $\mathrm{B}$ (Fig. 7d). We also found that isoform $\mathrm{C}$ is dominant in pluripotent stem cells and isoform $\mathrm{B}$ is present more in terminally differentiated cells and cancer cells (Supplementary Fig. 7c). The RNA-Seq data showed that the exon 6 and 9 skipping is rare, suggesting that isoform $C$ is the dominant isoform in hESCs (Supplementary Fig. 7d). To provide more functional validation of this isoform change, we assessed rescue of ZNF207 KD via overexpression of three ZNF207 isoforms respectively. Only forced expression of isoform $\mathrm{C}$ was able to restore the ZNF207 KD phenotype in hESCs (Fig. 7e, Supplementary Fig. 7e); overexpression of isoform A and B had no rescue effect. Gene expression analysis further demonstrated that isoform $\mathrm{C}$ rescued the differentiation by reactivating the expression of downstream targets, such as SALL4 and OTX2, through its transcription factor activity (Fig. 7f). These results 
a
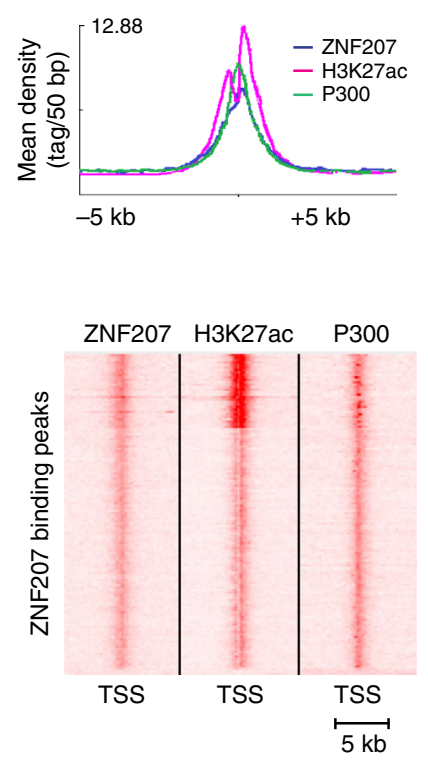

b

Fold enrichment 0

Mitotic cell cycle checkpoint

Stem cell maintenance

Cell cycle check point

Cell cycle phase transition

Mitotic cell cycle

Regulation of metabolic process

$p$-value $0.00 \mathrm{E}+00$

$1.00 \mathrm{E}-03$

$2.00 \mathrm{E}-03$

C

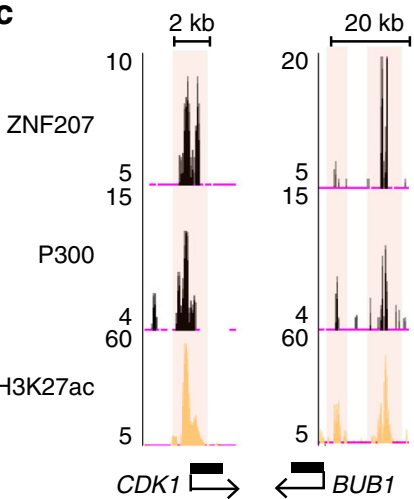

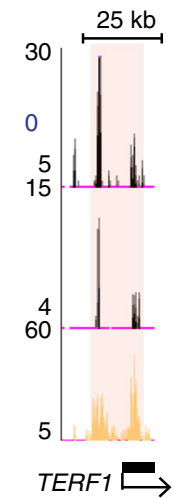

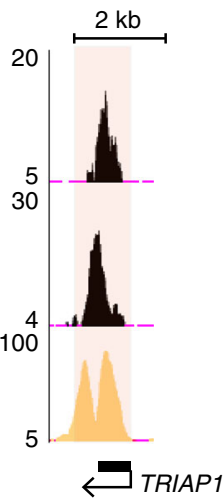

d Upregulated
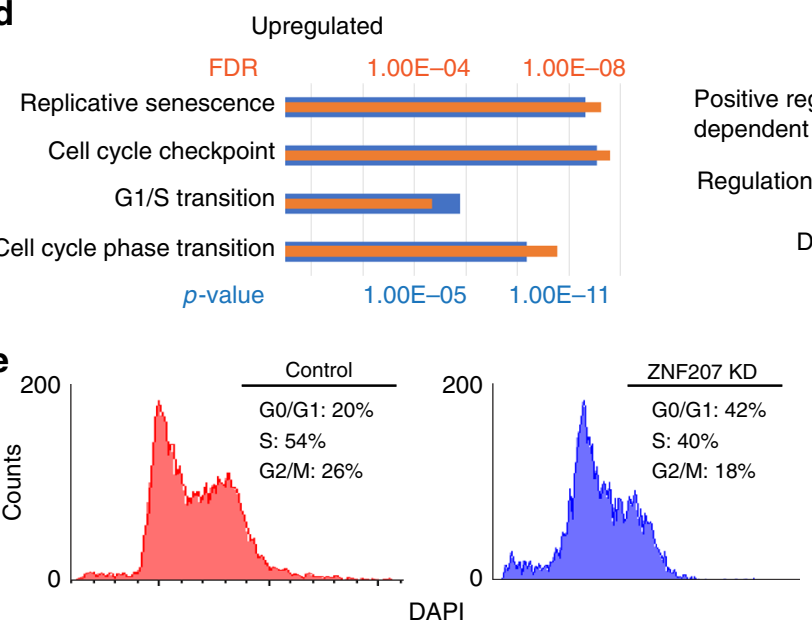

h
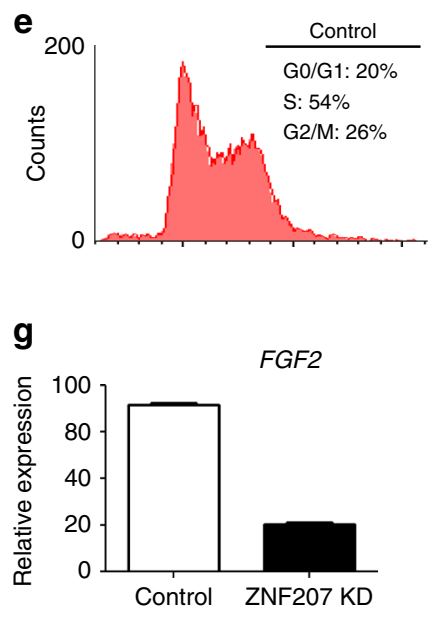

0

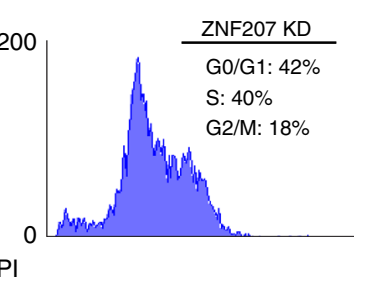

${ }_{5}^{15}$
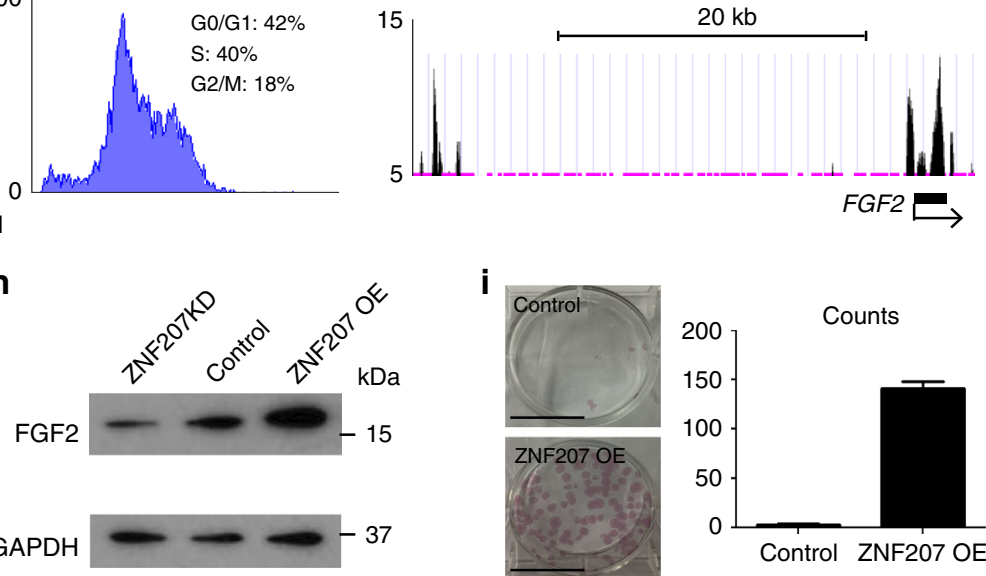

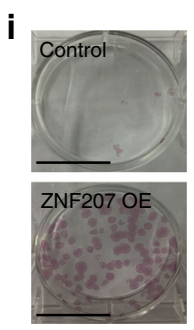

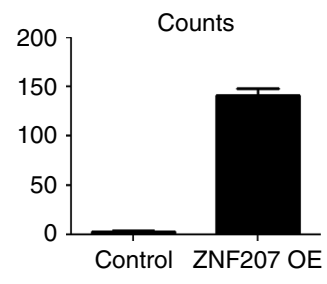

Fig. 5 ZNF207 co-localizes with enhancer markers to promote cell cycle of hESCs. a Top: Composite plot shows ZNF207, p300 (GSM1003513) and H3K27ac (GSM733718) ChIP-Seq signals are enriched and overlapped at TSSs. Bottom: Heatmap illustrates genome-wide association of ZNF207 with p300 and H3K27ac-binding sites. b Gene ontology analysis of genes that are co-bound by ZNF207, p300 and H3K27ac. Blue bars represent fold enrichment and orange bars represent $p$-value. $p$-value is calculated using hypergeometric distribution. c ChIP-Seq tracks show co-localization of ZNF207, P300, and H3K27ac at the regulatory sequences of cell cycle genes. The scale bars indicate the size of the chromosome. The light pink boxes highlight the co-bound regions. d Gene ontology analysis of cell cycle genes that are differentially expressed in ZNF207 KD cells. Blue bars represent $p$-values and orange bars represent FDR (false discovery rate). $p$-value is calculated using hypergeometric distribution. e Cell cycle progressions determined by flow cytometry. Histogram plot of flow cytometry analysis of control cells (red) and ZNF207 KD cells (blue). The percentage of cells that are in each phase of mitosis is shown. $\mathbf{f}$ ChIP-Seq tracks show binding of ZNF2O7 at promoter and enhancer regions of FGF2 gene. $\mathbf{g}$ The expression of FGF2 in control and ZNF207 KD cells. $\mathbf{h}$ Western blot to detect the protein level of FGF2 in KD, control, and OE cells. GAPDH is shown as the loading control. i Clonogenic assay to show the self-renewal ability of control and ZNF207 OE cells in E7 media with $20 \mathrm{ng} / \mathrm{ml}$ bFGF. Scale bars represent $17.5 \mathrm{~mm}$. The counts of colonies are shown on the right. Data are presented as the mean \pm SEM and are derived from three independent experiments 
a

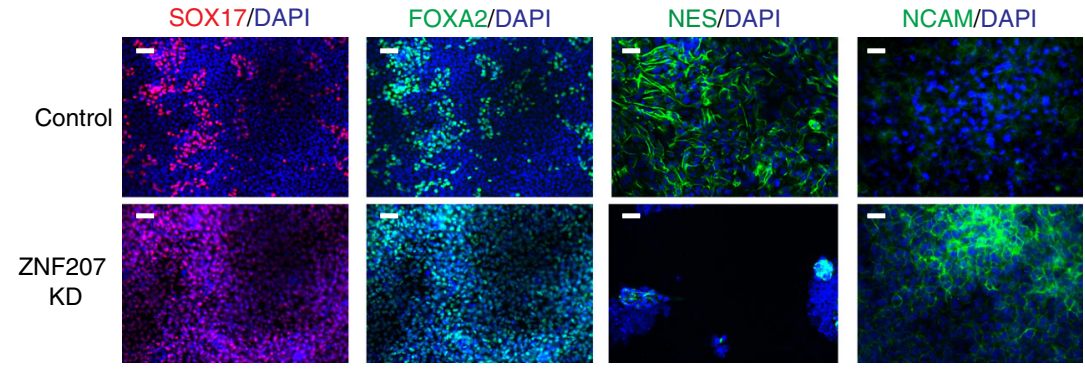

b
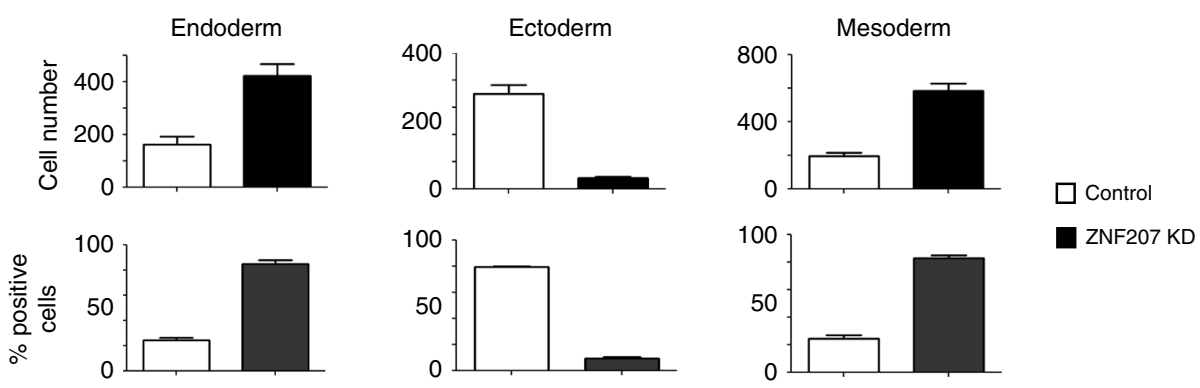

C
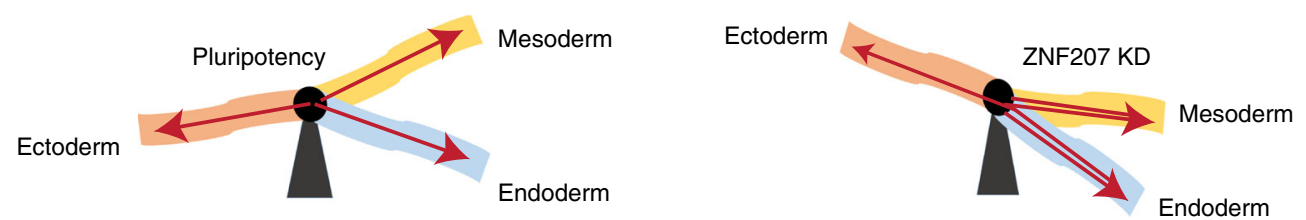

d
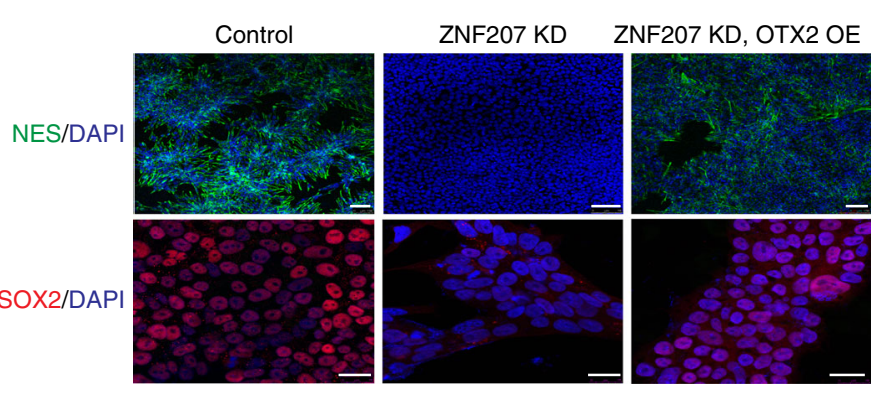

e

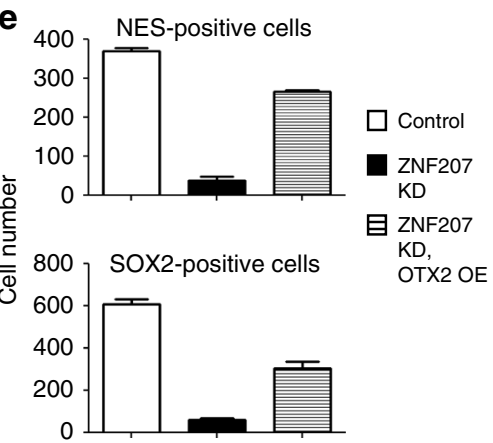

$\mathbf{f}$
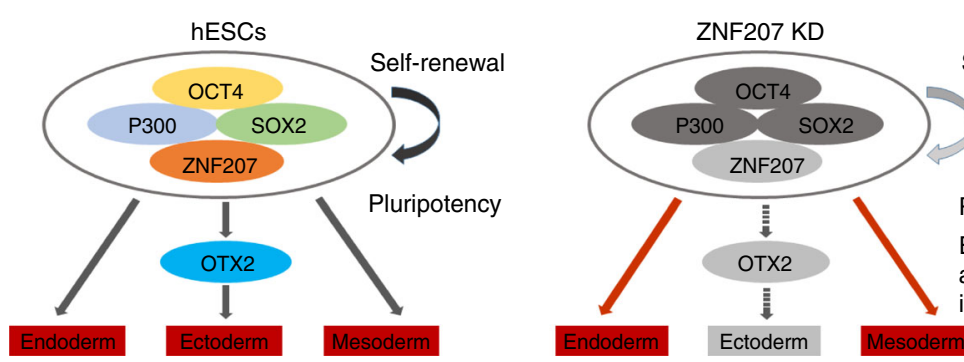

Self-renewal Reduced

Pluripotency

Enhanced potential to endoderm and mesoderm;

impaired potential to ectoderm

Fig. 6 ZNF207 works upstream of OTX2 in hESCs to govern specification to ectoderm. a Immunofluorescence staining of control and ZNF207 KD hESCs for lineage markers. Scale bars represent $50 \mu \mathrm{m}$. b Counts and percentage of cells that were stained positive for each lineage. c KD of ZNF207 disrupted pluripotency of hESCs. HESCs have equal potential to differentiate into any of the three lineages, while KD of ZNF207 tilts the potential to endoderm and mesoderm. The potential to differentiate into ectoderm is significantly impaired by KD of ZNF207. d Immunofluorescence staining of control, ZNF207 KD and ZNF207 KD, OTX2 OE hESCs for ectodermal proteins. Top: Scale bars represent $100 \mu \mathrm{m}$; Bottom: Scale bars represent $25 \mu \mathrm{m}$. e Counts of cells that were stained positive for ectodermal proteins. f ZNF207-centered transcriptional network to regulate self-renewal and pluripotency in hESCs. ZNF207 works with master transcription factors and co-factors, such as OCT4, SOX2, and P300, to form the core transcriptional network in hESCs. They bind together at pluripotency, development, and cell cycle-related genes to govern self-renewal and pluripotency. OTX2 is targeted and regulated by ZNF207 to ensure the developmental potential towards ectoderm. Once ZNF207 is knocked down, the expression of OCT4, SOX2, and P300 is reduced. The level of cell cycle-related genes has been changed, which leads to reduced ability of self-renewal. The expression of OTX2, which is downstream of ZNF207, is also downregulated, which impedes the differentiation potential towards ectoderm. In the meantime, the potential to endoderm and mesoderm is enhanced. Data are presented as the mean \pm SEM and are derived from three independent experiments 
a

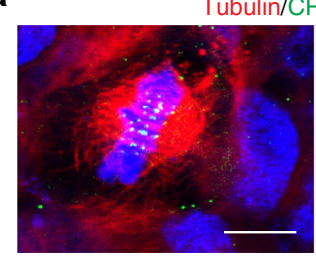

Control

C

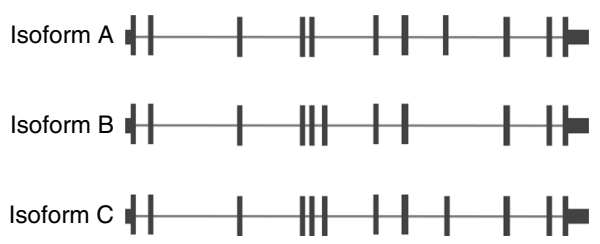

e
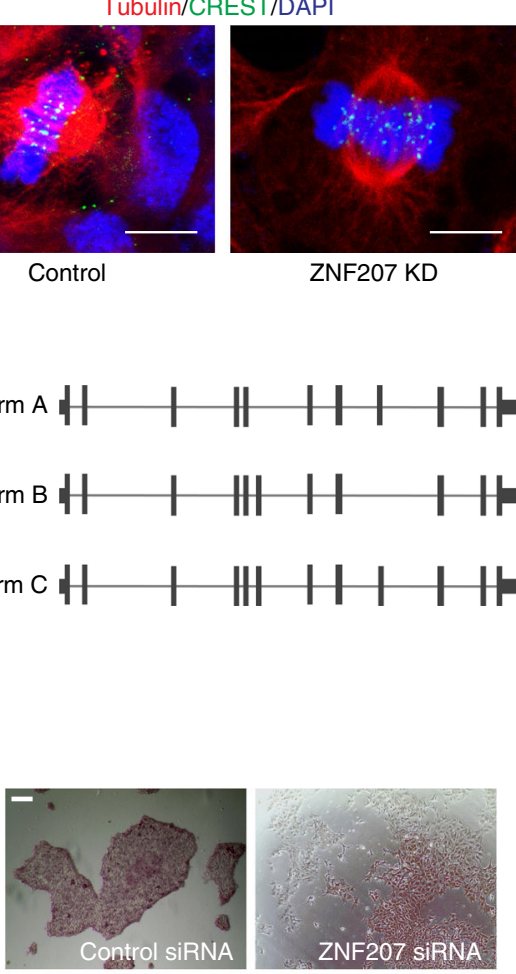

ZNF207 KD

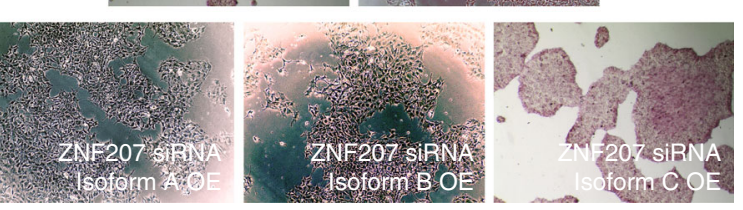

b

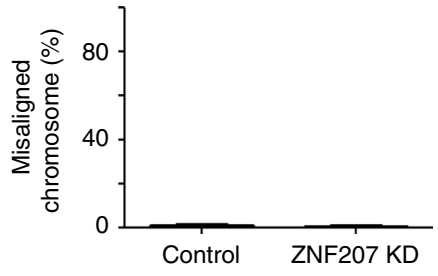

d

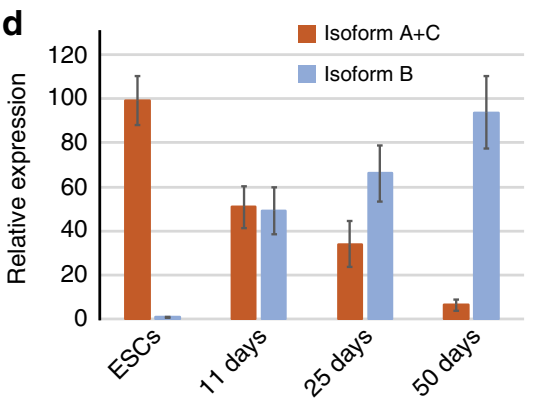

f

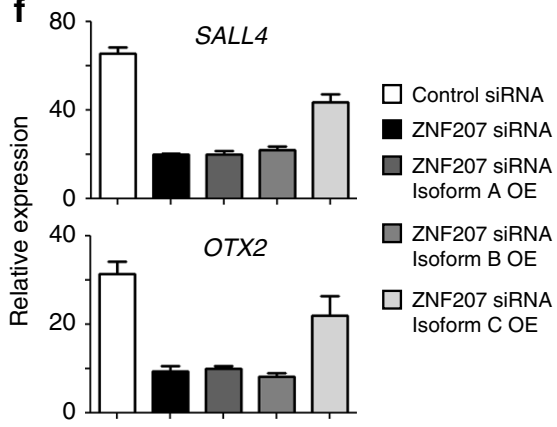

g

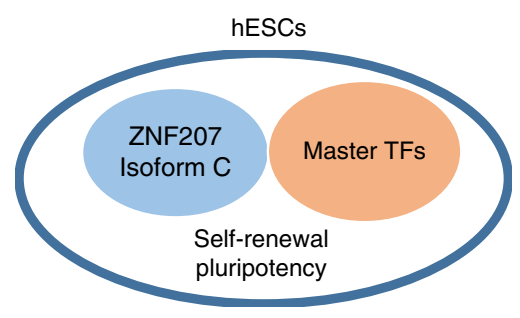

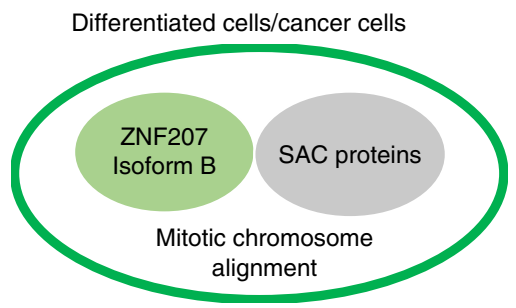

Fig. 7 ZNF207 switches isoforms during cell differentiation. a Confocal images of mitotic cells with aligned chromosomes. 100 mitotic cells were measured for each experiment and condition. Centromeres were detected by the CREST serum. Scale bar, $5 \mu$ m. $\mathbf{b}$ Counts of mitotic cells with misaligned chromosomes in control and ZNF207 KD cells. c Schematic representation of the three alternative splice isoforms of ZNF207. d RT-qPCR analysis to test the expression level of different isoforms during induced neuronal differentiation from hESCs. e Alkaline phosphatase staining of cells in different conditions. Scale bars represent $50 \mu \mathrm{m}$. $\mathbf{f}$ RT-qPCR analysis to test the expression level of SALL4 and OTX2. Data are presented as the mean \pm SEM and are derived from three independent experiments. $\mathbf{g}$ The model of ZNF207 isoform change in hESCs and differentiated/cancer cells. Data are presented as the mean \pm SEM and are derived from three independent experiments

indicated that ZNF207 switches isoforms in different cell types and individual isoform play distinct roles in the cell. Isoform $\mathrm{C}$ of ZNF207 is dominantly present in hESCs and is the functional isoform of ZNF207 that interacts with master TFs and co-factors to function as a TF to control the transcription of key genes in order to maintain self-renewal and pluripotency of hESCs. In differentiated cells and cancer cells, isoform B of ZNF207 interacts with spindle assembly checkpoint (SAC) proteins to regulate mitotic chromosome alignment (Fig. $7 \mathrm{~g}$ ).

\section{Discussion}

The OCT4 TF is required to induce and maintain self-renewal and pluripotency in pluripotent stem cells ${ }^{1,42}$. In this study, endogenous regulation of OCT4 in hESCs was probed through locus-specific proteomics and ZNF207 was identified as a critical regulator for self-renewal and pluripotency and a key driver of differentiation of hESCs to ectoderm. On the one hand, ZNF207 works with master TFs as a guardian of pluripotency and selfrenewal in hESCs controlling the expression of pluripotency genes such as OCT4, P300, SALL4, and PRDM14, as well as selfrenewal genes such as $C D K 1, B U B 1$, and TERF. Dysregulation of any of these genes is known to induce loss of hESC pluripotency and self-renewal. Importantly, data suggests that ZNF207 functions to drive initial differentiation, especially the differentiation potential towards ectoderm. As indicated in the Waddington's canal $^{43}$, wild-type hESCs have equal potential to differentiate into any of the three primary germ layers if provided appropriate 
conditions. However, KD of ZNF207 tilts the potential of differentiation towards endoderm and mesoderm; Their potential to become ectoderm has been significantly impaired (Fig. 6c). It has been proposed that pluripotency TFs may not work as inhibitory TFs that cooperatively block differentiation to all lineages but instead may modulate lineage specifiers that direct commitment to different lineages ${ }^{35-37}$. For example, Nanog enhances the ability to differentiate into definitive endoderm ${ }^{44}$ and SALL4 is required for specification of trophoblasts ${ }^{45}$. Similarly, Sox2 and Otx2 are implicated in ectoderm specification ${ }^{46}$. We add ZNF207 to the membership of the pluripotency/ectoderm specification factor cascade. ZNF207 promotes ectoderm differentiation by activating early neuronal genes, including $O T X 2$, a gene required for ESC-derived neural precursors to differentiate into telencephalon and mesencephalon ${ }^{47,48}$. The ability of ZNF207 to activate and maintain OTX2 expression while promoting hESC self-renewal and pluripotency is indicative of an early driver of hESC differentiation to ectoderm.

These studies report that in hESCs, isoform A and C of ZNF207 are abundant and isoform $\mathrm{C}$ functions as a transcription factor to maintain the core transcriptional network. Once hESCs differentiate, ZNF207 switches from isoform A and C to isoform B. Our data, together with previous reports, suggests that this change of isoform switches the function of ZNF207 from a critical transcription regulator to a SAC regulator during mitosis. We hypothesize that switching isoforms may be a common underlying mechanism for TFs to play distinct roles in various cell types during development. Notably, during differentiation, 489 genes in hESCs may change isoforms including 33 which are classified as $\mathrm{TFs}^{41}$.

In conclusion, these studies demonstrated that a locus-specific proteomics approach is able to identify known OCT4 regulators, such as OCT4, SOX2, SALL4, and HDAC2, and also identify previously unknown proteins that regulate OCT4 expression. Findings illuminate specific aspects of transcriptional regulation in pluripotent stem cells while expanding our understanding of the intrinsic transcriptional network that controls identity of hESCs and promotes differentiation to one of three primary germ layers in human development, the ectoderm. Furthermore, given the expression of ZNF207 in human embryos, it is of interest to investigate the roles of ZNF207 in early human embryo development at pre-implantation and/or post-implantation stages.

\section{Methods}

Cell culture. Human ES cell-line (H9, WiCell) was cultured feeder-free on matrigel matrix (BD Biosciences) and maintained in mTeSR media (STEMCELL Technologies $)^{49}$. In the bFGF clonogenic experiments, hESCs were culture in Essential 6 medium (ThermoFisher; A1516401) with addition of $2 \mathrm{ng} / \mathrm{ml}$ TGF $\beta$ (R\&D systems) and $20 \mathrm{ng} / \mathrm{ml}$ bFGF (Pepro Tech). Naive hPSCs were kindly provided by Dr. Sarita P. Panula ${ }^{50}$ and cultured in $\mathrm{t} 2 \mathrm{i} / \mathrm{L}+\mathrm{PKCi}$ conditions in a 1:1 mixture of DMEM/F12 and Neurobasal, 0.5x N2-supplement, 0.5x B27-supplement, 1x nonessential amino acids, $2 \mathrm{mM}$ L-Glutamine, 1x Penicillin/Streptomycin (all from ThermoFisher Scientific), $0.1 \mathrm{mM} \beta$-mercaptoethanol (Sigma-Aldrich), $1 \mu \mathrm{M}$ PD0325901, $1 \mu \mathrm{M}$ CHIR99021, $20 \mathrm{ng} / \mathrm{ml}$ human LIF (all from WT-MRC Cambridge Stem Cell Institute), and $2 \mu \mathrm{M}$ Gö6983 (PKCi; Tocris) on a MEF-layer seeded at a density of $2 \times 10^{6}$ cells per 6 -well plate ${ }^{25}$.

\section{Construction of TALE plasmid. Modified TALE constructs targeting OCT4} proximal enhancer were designed and assembled based on the backbone of Daniel Voytas lab golden gate talen assembly 2.0 (Addgene, Kit \#1000000024). A control TALE protein targeting green fluorescence protein (GFP) was also designed and assembled. For hESC overexpression, the CMV promoter was replaced with human EF1a promoter. For affinity enrichment of chromatin from the proximal enhancer region of the OCT4 gene in hESCs, TALE proteins were designed to bind genomic sequences of OCT4 proximal enhancer (Supplementary Table 1). The TALE protein was designed as a truncation that lacked Fok I nuclease domain. The 3xFLAG tag was added to the $\mathrm{N}$-terminus and maxGFP was added to the $\mathrm{C}$-terminus for visualization. Lentiviral version of modified TALE plasmid was constructed by subcloning the TALE ORF to the lentiviral overexpression vector pLex307 (Addgene \#41392).
Transfection. Transfection of dTALE construct in hESCs was performed using Fugene 6 (Promega) according to manufacturer's instructions. Puromycin (Sigma) selection was introduced 1 day after transfection at $2.0 \mu \mathrm{g} / \mathrm{ml}$.

Virus generation. Lentiviruses were produced in HEK293T cells (ATCC, VA) by cotransfection with two helper plasmids (pVSVG and pDelta8.9: $12 \mu \mathrm{g}$ of lentivira vector DNA and $6 \mathrm{mg}$ of each of the helper plasmid DNA per $75 \mathrm{~cm}^{2}$ culture area) using Lipofectamine 2000 (Invitrogen) $^{51}$. Lentiviruses were harvested with the medium $46 \mathrm{~h}$ after transfection, pelleted by centrifugation $(49,000 \times \mathrm{g}$ for $90 \mathrm{~min})$, resuspended in MEM, aliquoted, and snap-frozen in liquid $\mathrm{N}_{2}$. Only virus preparations with $>90 \%$ infection efficiency as assessed by EGFP expression or puromycin resistance were used for experiments. The sequence of ZNF207 OE construct is shown in Supplementary Table 4.

Viral transduction. Undifferentiated $\mathrm{H} 9$ cells were transduced on Matrigel-coated plates with dTALE or plex307-ZNF207 (isoform C) or plex307-OTX2 overexpression vectors along with viral supernatant supplemented with $8 \mu \mathrm{g} / \mathrm{ml}$ polybrene (Sigma-Aldrich). After $6 \mathrm{~h}$, Virus supernatants were washed off, and fresh mTESR 1 medium was added. Subsequently, cells were selected with $2 \mu \mathrm{g} / \mathrm{ml}$ puromycin (Invitrogen) for 4 days in mTESR1 media.

Co-immunoprecipitation. Transfected cells were lysed in cell lysis buffer $(50 \mathrm{mM}$ Tris- $\mathrm{HCl} \mathrm{pH} 8.0,150 \mathrm{mM} \mathrm{NaCl}, 1 \mathrm{mM}$ EDTA, $1 \%$ NP40, 10\% glycerol with protease inhibitor cocktail) for $1 \mathrm{~h}$. Whole-cell extracts were collected and precleared. Beads coated with FLAG antibody (CST \#2368), OCT4 antibody (sc-8628, Santa Cruz Biotechnology), and ZNF207 antibody (PA5-30641, ThermoFisher Scientific) were incubated with precleared whole-cell extracts at $4{ }^{\circ} \mathrm{C}$ overnight. The beads were washed with cell lysis buffer four times. Finally, the beads were boiled in $2 x$ sample buffer for $10 \mathrm{~min}$. The eluents were analyzed by Western blot.

Mass spectrometry. Briefly, $10^{11}$ OCT4 targeting TALE and control GFP targeting TALE transduced hESC cells were crosslinked by $1 \%$ formaldehyde, then collected, and lysed by ChIP lysis buffer. Genomic DNA was sonicated to $1 \mathrm{~kb}$. Proteins complexes co-enriching with the TALE-FLAG or proteins non-specifically binding to the anti-FLAG Dynabeads (Invitrogen) were reverse crosslinked by incubation at $65^{\circ} \mathrm{C}$ for $6 \mathrm{~h}$. Proteins were purified by acetone precipitation and then resuspended in Tris-HCl buffer. A fraction of the protein samples was then resolved by SDS-PAGE/Silver-staining. The protein sample was concentrated by using a Microcon YM-10 column (Millipore) according to the manufacturer's protocol. Approximately $10 \mu \mathrm{l}$ of sample was obtained from the filter device. A $1 \mu \mathrm{l}$ portion of $100 \mathrm{mmol} / \mathrm{l}$ DTT (Sigma) was added, and the resulting mixture was incubated at $45^{\circ} \mathrm{C}$ for $30 \mathrm{~min}$. A $1 \mu \mathrm{l}$ portion of trypsin (Sigma) was added afterward at $37^{\circ} \mathrm{C}$. The overnight digestion and reduction reaction was terminated by adding $0.1 \mu \mathrm{l}$ of TFA to the digest. LC/MS/MS was performed on an 1100 series capillary LC system (Agilent Technologies) coupled to an LTQ-Orbitrap mass spectrometer (Thermo) operating in positive mode and equipped with a nanospray source. All MS/MS spectra were analyzed by using the SEQUEST algorithm, incorporated in Bioworks software, version 3.1 SR1 (Thermo Finnigan). Peptide fragment lists were generated and submitted to the Swiss-Prot database. Two independent experiments were performed. LC/MS/MS was performed by Caprion Biosciences, Inc.

Chromatin immunoprecipitation and ChIP-Seq. ChIP assays were carried out as described $^{52}$. Briefly, cells were crosslinked with $1 \%$ formaldehyde for $10 \mathrm{~min}$ at room temperature, followed by the addition of $0.2 \mathrm{M}$ glycine to inactivate the formaldehyde. Cells were then lysed to obtain chromatin extracts, which were sonicated to obtain DNA fragments with an average size of 300-500 bp. The resulting chromatin extracts were immunoprecipitated by antibodies immobilized on Protein-G beads. The antibody information is shown in Supplementary Table 10. Relative occupancy values (fold enrichment) were calculated by determining the apparent immunoprecipitation efficiency and normalized to the level observed at a control region, which was defined as 1.0. All ChIP experiments were repeated at least three times and data are presented as the mean \pm SEM. Primer sequences are available in Supplementary Table 5. For ChIP-Seq, Illumina HiSeq $2 \times 60 \mathrm{bp}$ paired-end reads were used for sequencing.

Motif analysis. Motif analysis was performed using MEME-ChIP suite with default parameters ${ }^{53}$. The motifs for ZNF207 were searched in TRANSFAC and JASPER databases to find transcription factors with similar consensus sequences ${ }^{54,55}$.

Electrophoretic mobility shift assays. Recombinant human ZNF207 protein was

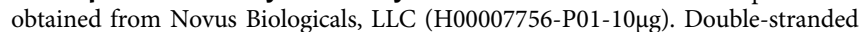
DNA oligonucleotides labeled with biotin at the 5 ' termini of the sense strands (Integrated DNA Technologies, Inc) were annealed with reverse strands in annealing buffer (10 mM Tris-HCl, $\mathrm{pH} 8.0,50 \mathrm{mM} \mathrm{NaCl}, 1 \mathrm{mM}$ EDTA) and purified with an agarose gel DNA extraction kit (Qiagen). The sense strand sequence is shown in Fig. 3d. EMSA was performed in a 10- $\mu$ l reaction mixture 
containing $10 \mathrm{mM}$ HEPES, pH7.5, $10 \mathrm{mM} \mathrm{KCl,} 10 \mathrm{mM} \mathrm{MgCl}, 1 \mathrm{mM}$ DTT, $1 \mathrm{mM}$ EDTA, $10 \%$ glycerol, $3 \mathrm{ng}$ of biotin-labeled oligonucleotide, $1 \mu \mathrm{g}$ of poly(dI-dC) (Amersham) and $100 \mathrm{ng}$ recombinant ZNF207 protein (isoform C). Binding reaction mixtures were incubated for $10 \mathrm{~min}$ at room temperature and then subjected to electrophoresis on pre-run 5\% native PAGE gels in $0.5 \mathrm{x}$ TBE buffer. Gels were transferred to Biodyne B nylon membranes (Pierce Biotechnologies) and detected with LightShift Chemiluminescent EMSA kit (Pierce Biotechnologies).

Knockdown by siRNA. Three siRNAs against each candidate gene were purchased from Invitrogen ${ }^{\mathrm{TM}}$ Silencer ${ }^{\mathrm{TM}}$ Pre-designed siRNAs. The sequence of siRNA is listed in Supplementary Table 5, 6. siRNA transfection was performed with Lipofectamine RNAiMAX (Invitrogen). In brief, for each well of 12 -well plate, 6 pmol of siRNA were mixed with $1 \mu$ l of Lipofectamine RNAiMAX in 100ul of OptiMEM medium (Invitrogen). The mixture was added to $1-3 \times 10^{5}$ cells with $1 \mathrm{ml}$ of cell culture medium without antibiotics. Twenty-four hours after transfection, the cell culture medium was replaced with fresh medium. Four days after transfection, cells were imaged or harvested for RNA extraction.

RNA extraction, reverse transcription, and quantitative real-time PCR. Tota RNA was extracted using TRIzol Reagent (Invitrogen) and purified with the RNeasy Mini Kit (Qiagen). Reverse transcription was performed using SuperScript II Kit (Invitrogen). Quantitative PCR analyses were performed in real time using an ABI PRISM 7900 sequence detection system and SYBR green master mix (Life Technologies). The primer information is provided in Supplementary Table 8. The levels of the transcripts were normalized against control empty vector transfection. Data are presented as the mean \pm SEM and derived from three independent experiments.

RNA-Seq and differential expression analysis. Total RNA was extracted using Acruturus PicoPure RNA Isolation Kit (Life Technologies). RNA quality was determined with Bioanalyzer 2100 (Agilent). Sequencing libraries were constructed by SMARTer universal low input RNA Kit (Clonetech) according to the manufacturer's instructions. DNA library samples were submitted to the Stanford Genomics Facility and 100-base paired-end high-throughput sequencing was performed. All sequenced libraries were mapped to the human genome using TopHat and Cufflink $k^{56,57}$ with default parameter setup. Differential expression was analyzed using StrandNGS (AvadisNGS).

Gene ontology analysis. The Gene Ontology (GO) enrichment was done using GREAT analysis, with default parameters ${ }^{58}$. For Stringent analysis, we set-up the gene association rule to associate TSS only in $15 \mathrm{~KB}$ distance.

Immunofluorescence analysis. Cells were fixed with $4 \%$ paraformaldehyde at room temperature for $20 \mathrm{~min}$, permeabilized with $0.3 \%$ Triton X-100 in PBS (PBST) for $10 \mathrm{~min}$, and blocked for $45 \mathrm{~min}$ at room temperature in PBST containing 5\% normal donkey serum (Jackson ImmunoResearch Laboratories). Primary antibodies were diluted in blocking solution and incubated at $4{ }^{\circ} \mathrm{C}$ overnight. Appropriate Alexa Fluor 488 or 594-conjugated secondary antibodies (Jackson ImmunoResearch Laboratories) were diluted in PBS containing $0.1 \%$ bovine serum albumin (BSA) and incubated at room temperature for $1 \mathrm{~h}$. Finally, $1 \mu \mathrm{g} / \mathrm{ml}$ DAPI was used for nuclear staining. The information of primary and secondary antibodies used in this study are listed in Supplementary Table 9 .

Western blot analysis. The cells were washed with cold PBS and then scraped from the plate in $1 \mathrm{ml}$ PBS plus $2 \times$ protease inhibitors (Complete Mini, Roche) and transferred immediately to a $1.5 \mathrm{ml}$ tube on ice. The cell suspension was then spun at 5000 r.p.m. in a microcentrifuge for $3 \mathrm{~min}$ and the supernatant was discarded. The cell pellet was resuspended with $200 \mu \mathrm{l}$ RIPA buffer $(50 \mathrm{mM}$ Tris, $150 \mathrm{mM}$ $\mathrm{NaCl}, 0.5 \%$ Sodium deoxycholate, $1 \% \mathrm{NP}-40,0.1 \% \mathrm{SDS}, \mathrm{pH} 8$ ) plus $2 \times$ protease inhibitors (Complete Mini, Roche). The cell pellet suspension was pipetted rigorously at least 10 times, then vortexed for $30 \mathrm{~s}$. The suspension was again spun down for $3 \mathrm{~min}$ at the same speed. The supernatant was denatured in 4XNuPAGE LDS sample buffer (ThermoFisher) at $95^{\circ} \mathrm{C}$ for $5 \mathrm{~min}$, then loaded onto a precast NuPAGE $10 \%$ Bis-Tris midi protein gel (Invitrogen). The SDS-PAGE gels were run at 150 volts for $1 \mathrm{~h}$ and transferred to a PVDF membrane by iBlot transfer system (Invitrogen). Transferred blots were blocked in $5 \%$ non-fat milk for $1 \mathrm{~h}$ at room temperature. The blot was subjected to $1 \mathrm{~h}$ of primary antibody incubation, followed by two quick rinses and three washes for $5 \mathrm{~min}$ in TBST (TBS, pH 7.4 with $0.1 \%$ Tween-20). Secondary antibody incubation had the same duration and washes. ECL + (Amersham) was used to detect the HRP signal and the western blot image was collected using Chemidoc XRS (Bio-Rad). Uncropped western blots are shown in Supplementary Fig. 8. The information for specific antibodies are listed in Supplementary Table 9.

Luciferase assay for enhancer activity. Enhancer sequences were generated by PCR of human genomic DNA discovered for gene OCT4 and then cloned into
pGL4.28 (Promega) with restricted enzymes KpnI and BmtI (NEB). The minima promoter pGL4.28 is used as negative control. Luciferase activity was measured using dual-luciferase reporter assay system (Promega) as described in the manufacturer's Manu.

FACS and flow cytometry. Cells may be fixed with $4 \%$ paraformaldehyde and stained with DAPI at room temperature for $15 \mathrm{~min}$. Then, cells were dissociated in $0.25 \%$ trypsin--EDTA (Gibco BRL) at $37^{\circ} \mathrm{C}$ for $5 \mathrm{~min}$ and collected by centrifugation at 200×g in an Eppendorf 5702R centrifuge. Then the cells were passed through the 70uM strainers (BD Biosciences) to make sure they were digested as single cells before they were subject to the flow cytometry. The analysis was performed using LSRII (Becton Dickinson) and FlowJo software (Tree Star).

Differentiation of hESCs to three lineages. hESCs were differentiated using STEMdiffTM trilineage differentiation kit (\#05230, Stem Cell Technology) based on manufacture's protocol.

\section{Highlights}

- Genome-wide, unbiased locus-specific proteomics identifies endogenous regulators of OCT4 in human embryonic stem cells (hESCs).

- ZNF207 interacts with master TFs to maintain hESC self-renewal and pluripotency.

- ZNF207 enhances reprogramming efficiency through induction of endogenous OCT4 expression.

- ZNF207 promotes self-renewal of hESCs.

- ZNF207 controls commitment to ectoderm.

- Different isoforms and functions of ZNF207 occur in various cell types.

\section{Data availability}

The ChIP-seq data and RNA-seq data of ZNF207 in hESCs have been deposited in the NCBI Gene Expression Omnibus database (http://www.ncbi.nlm.nih.gov/geo/) under the accession code GSE118638. The mass spectrometry proteomics data have been deposited to the ProteomeXchange Consortium (http://proteomecentral.proteomexchange.org) via the PRIDE partner repository with the data set identifier PXD010963. All other data supporting the findings of this study are available from the corresponding author upon reasonable request.

Received: 21 September 2017 Accepted: 21 September 2018 Published online: 22 October 2018

\section{References}

1. Boyer, L. A. et al. Core transcriptional regulatory circuitry in human embryonic stem cells. Cell 122, 947-956 (2005).

2. Chia, N.-Y. Y. et al. A genome-wide RNAi screen reveals determinants of human embryonic stem cell identity. Nature 468, 316-320 (2010).

3. Tsankov, A. M. et al. Transcription factor binding dynamics during human ES cell differentiation. Nature 518, 344-349 (2015).

4. Van den Berg, D. L. et al. An Oct4-centered protein interaction network in embryonic stem cells. Cell Stem Cell 6, 369-381 (2010).

5. Young, R. A. Control of the embryonic stem cell state. Cell 144, 940-954 (2011).

6. Niwa, H., Miyazaki, J. \& Smith, A. G. Quantitative expression of Oct-3/4 defines differentiation, dedifferentiation or self-renewal of ES cells. Nat. Genet. 24, 372-376 (2000)

7. Byrum, S. D., Raman, A., Taverna, S. D. \& Tackett, A. J. ChAP-MS: a method for identification of proteins and histone posttranslational modifications at a single genomic locus. Cell Rep. 2, 198-205 (2012).

8. Byrum, S. D., Taverna, S. D. \& Tackett, A. J. Purification of a specific native genomic locus for proteomic analysis. Nucleic Acids Res. 41, e195 (2013).

9. Déjardin, J. \& Kingston, R. E. Purification of proteins associated with specific genomic Loci. Cell 136, 175-186 (2009).

10. Hoshino, A. \& Fujii, H. Insertional chromatin immunoprecipitation: a method for isolating specific genomic regions. J. Biosci. Bioeng. 108, 446-449 (2009).

11. Ide, S. \& Dejardin, J. End-targeting proteomics of isolated chromatin segments of a mammalian ribosomal RNA gene promoter. Nat. Commun. 6, 6674 (2015).

12. Pourfarzad, F. et al. Locus-specific proteomics by TChP: targeted chromatin purification. Cell Rep. 4, 589-600 (2013).

13. Soldi, M. \& Bonaldi, T. The ChroP approach combines ChIP and mass spectrometry to dissect locus-specific proteomic landscapes of chromatin. J. Vis. Exp. 86, 51220 (2013). 
14. Waldrip, Z. J. et al. A CRISPR-based approach for proteomic analysis of a single genomic locus. Epigenetics 9, 1207-1211 (2014).

15. Minucci, S. et al. Retinoic acid-mediated down-regulation of Oct $3 / 4$ coincides with the loss of promoter occupancy in vivo. EMBO J. 15, 888-899 (1996).

16. Okazawa, $\mathrm{H}$. et al. The oct3 gene, a gene for an embryonic transcription factor, is controlled by a retinoic acid repressible enhancer. EMBO J. 10, 2997-3005 (1991).

17. Yeom, Y. I. et al. Germline regulatory element of Oct-4 specific for the totipotent cycle of embryonal cells. Development 122, 881-894 (1996).

18. Gafni, O. et al. Derivation of novel human ground state naive pluripotent stem cells. Nature 504, 282-286 (2013).

19. Munoz Descalzo, S. et al. A competitive protein interaction network buffers Oct4-mediated differentiation to promote pluripotency in embryonic stem cells. Mol. Syst. Biol. 9, 694 (2013).

20. Wang, J. et al. A protein interaction network for pluripotency of embryonic stem cells. Nature 444, 364-368 (2006).

21. Jamaladdin, S. et al. Histone deacetylase (HDAC) 1 and 2 are essential for accurate cell division and the pluripotency of embryonic stem cells. Proc. Natl Acad. Sci. USA 111, 9840-9845 (2014).

22. Casolari, J. M. et al. Genome-wide localization of the nuclear transport machinery couples transcriptional status and nuclear organization. Cell 117, 427-439 (2004).

23. Rodriguez, R. T. et al. Manipulation of OCT4 levels in human embryonic stem cells results in induction of differential cell types. Exp. Biol. Med. 232, 1368-1380 (2007).

24. Nakamura, T. et al. A developmental coordinate of pluripotency among mice, monkeys and humans. Nature 537, 57-62 (2016).

25. Takashima, Y. et al. Resetting transcription factor control circuitry toward ground-state pluripotency in human. Cell 158, 1254-1269 (2014).

26. Yan, L. et al. Single-cell RNA-Seq profiling of human preimplantation embryos and embryonic stem cells. Nat. Struct. Mol. Biol. 20, 1131-1139 (2013).

27. Petropoulos, $\mathrm{S}$. et al. Single-cell RNA-seq reveals lineage and X chromosome dynamics in human preimplantation embryos. Cell 165, 1012-1026 (2016).

28. Zeng, X. Human embryonic stem cells: mechanisms to escape replicative senescence? Stem Cell Rev. 3, 270-279 (2007).

29. White, J. \& Dalton, S. Cell cycle control of embryonic stem cells. Stem Cell Rev. 1, 131-138 (2005)

30. Neganova, I. \& Lako, M. G1 to $S$ phase cell cycle transition in somatic and embryonic stem cells. J. Anat. 213, 30-44 (2008)

31. Amit, M. et al. Clonally derived human embryonic stem cell lines maintain pluripotency and proliferative potential for prolonged periods of culture. Dev. Biol. 227, 271-278 (2000).

32. Levenstein, M. E. et al. Basic fibroblast growth factor support of human embryonic stem cell self-renewal. Stem Cells 24, 568-574 (2006).

33. $\mathrm{Xu}$, C. et al. Feeder-free growth of undifferentiated human embryonic stem cells. Nat. Biotechnol. 19, 971-974 (2001).

34. $\mathrm{Xu}, \mathrm{R}$. H. et al. Basic FGF and suppression of BMP signaling sustain undifferentiated proliferation of human ES cells. Nat. Methods 2, 185-190 (2005).

35. Loh, K. M. \& Lim, B. A precarious balance: pluripotency factors as lineage specifiers. Cell Stem Cell 8, 363-369 (2011).

36. Smith, A. Formative pluripotency: the executive phase in a developmental continuum. Development 144, 365-373 (2017).

37. Weidgang, C. E., Seufferlein, T., Kleger, A. \& Mueller, M. Pluripotency factors on their lineage move. Stem Cells Int. 2016, 6838253 (2016).

38. Han, M. et al. A systematic RNAi Screen reveals a novel role of a spindle assembly checkpoint protein BuGZ in synaptic transmission in C. elegans. Front. Mol. Neurosci. 10, 141 (2017).

39. Jiang, $\mathrm{H}$. et al. A microtubule-associated zinc finger protein, BuGZ, regulates mitotic chromosome alignment by ensuring Bub3 stability and kinetochore targeting. Dev. Cell. 28, 268-281 (2014).

40. Toledo, C. M. et al. BuGZ is required for Bub3 stability, Bub1 kinetochore function, and chromosome alignment. Dev. Cell. 28, 282-294 (2014).

41. Toh, C. X. et al. RNAi reveals phase-Specific global regulators of human somatic cell reprogramming. Cell Rep. 15, 2597-2607 (2016).

42. Takahashi, K. \& Yamanaka, S. Induction of pluripotent stem cells from mouse embryonic and adult fibroblast cultures by defined factors. Cell 126, 663-676 (2006).

43. Simpson, F. R. Organisers and genes. Eugen. Rev. 32, 89-89 (1940).

44. Teo, A. K. et al. Pluripotency factors regulate definitive endoderm specification through eomesodermin. Genes Dev. 25, 238-250 (2011).

45. Lim, C. Y. et al. Sall4 regulates distinct transcription circuitries in different blastocyst-derived stem cell lineages. Cell Stem Cell 3, 543-554 (2008).

46. Wang, Z., Oron, E., Nelson, B., Razis, S. \& Ivanova, N. Distinct lineage specification roles for NANOG, OCT4, and SOX2 in human embryonic stem cells. Cell Stem Cell 10, 440-454 (2012).
47. Liu, H. \& Zhang, S. C. Specification of neuronal and glial subtypes from human pluripotent stem cells. Cell Mol. Life Sci. 68, 3995-4008 (2011).

48. Acampora, D., Di Giovannantonio, L. G. \& Simeone, A. Otx2 is an intrinsic determinant of the embryonic stem cell state and is required for transition to a stable epiblast stem cell condition. Development 140, 43-55 (2013).

49. Ludwig, T. E. et al. Feeder-independent culture of human embryonic stem cells. Nat. Methods 3, 637-646 (2006).

50. Collier, A. J. et al. Comprehensive cell surface protein profiling identifies specific markers of human naive and primed pluripotent states. Cell Stem Cell 20, 874-890 (2017).

51. Salomonis, N. et al. Alternative splicing regulates mouse embryonic stem cell pluripotency and differentiation. Proc. Natl Acad. Sci. USA 107, 10514-10519 (2010).

52. Chen, $\mathrm{X}$. et al. Integration of external signaling pathways with the core transcriptional network in embryonic stem cells. Cell 133, 1106-1117 (2008).

53. Machanick, P. \& Bailey, T. L. MEME-ChIP: motif analysis of large DNA datasets. Bioinformatics 27, 1696-1697 (2011).

54. Sandelin, A. et al. Arrays of ultraconserved non-coding regions span the loci of key developmental genes in vertebrate genomes. BMC Genomics 5, 99 (2004).

55. Wingender, E., Dietze, P., Karas, H. \& Knuppel, R. TRANSFAC: a database on transcription factors and their DNA binding sites. Nucleic Acids Res. 24, 238-241 (1996).

56. Trapnell, C., Pachter, L. \& Salzberg, S. L. TopHat: discovering splice junctions with RNA-Seq. Bioinformatics 25, 1105-1111 (2009).

57. Trapnell, C. et al. Transcript assembly and quantification by RNA-Seq reveals unannotated transcripts and isoform switching during cell differentiation. Nat. Biotechnol. 28, 511-515 (2010).

58. McLean, C. Y. et al. GREAT improves functional interpretation of cisregulatory regions. Nat. Biotechnol. 28, 495-501 (2010).

\section{Acknowledgements}

The work was supported by Montana Board of Research and Commercialization Technology \#18-51-037. We thank Dr. Sarita P. Panula for providing the naive state stem cells. We thank Dr. Edward Dratz, Dr. Robert Usselman, and Dr. Amander Clark for helpful scientific discussions.

\section{Author contributions}

The study was conceived and designed by F.F., X.N., and R.R.P.; F.F. and X.N. performed most experiments (including proteomics, ChIP-seq, RNA-seq, knockdown, immunostaining, protein pull-down, EMSA, FACS, and luciferase reporter assay) and analyzed the data. B.A., J.C., and Z.C. performed immunostaining and counting for analyzing the function of ZNF207 in spindle assembly in hESCs and cancer cells. J.D.-D. and V.S. performed single-cell RNA-seq analysis for preimplantation human embryos. T.B. made viral vector based constructs for initial knockdown screening. The manuscript was written by F.F. and R.R.P. with input from the other authors.

\section{Additional information}

Supplementary Information accompanies this paper at https://doi.org/10.1038/s41467018-06908-5.

Competing interests: The authors declare no competing interests.

Reprints and permission information is available online at http://npg.nature.com/ reprintsandpermissions/

Publisher's note: Springer Nature remains neutral with regard to jurisdictional claims in published maps and institutional affiliations.

Open Access This article is licensed under a Creative Commons Attribution 4.0 International License, which permits use, sharing, adaptation, distribution and reproduction in any medium or format, as long as you give appropriate credit to the original author(s) and the source, provide a link to the Creative Commons license, and indicate if changes were made. The images or other third party material in this article are included in the article's Creative Commons license, unless indicated otherwise in a credit line to the material. If material is not included in the article's Creative Commons license and your intended use is not permitted by statutory regulation or exceeds the permitted use, you will need to obtain permission directly from the copyright holder. To view a copy of this license, visit http://creativecommons.org/ licenses/by/4.0/.

(C) The Author(s) 2018 